\title{
Simulation of two dimensional photonic band gaps
}

\author{
S. E. Dissanayake, K. A. I. L. Wijewardena Gamalath \\ Department of Physics, University of Colombo, Colombo 3, Sri Lanka \\ E-mail address: imalie@phys.cmb.ac.lk
}

\begin{abstract}
The plane wave expansion method was implemented in modelling and simulating the band structures of two dimensional photonic crystals with square, triangular and honeycomb lattices with circular, square and hexagonal dielectric rods and air holes. Complete band gaps were obtained for square lattice of square GaAs rods and honeycomb lattice of circular and hexagonal GaAs rods as well as triangular lattice of circular and hexagonal air holes in GaAs whereas square lattice of square or circular air holes in a dielectric medium $\varepsilon=18$ gave complete band gaps. The variation of these band gaps with dielectric contrast and filling factor gave the largest gaps for all configurations for a filling fraction around 0.1.The gap maps presented indicated that TM gaps are more favoured by dielectric rods while TE gaps are favoured by air holes. The geometrical gap maps operating at telecommunication wavelength $\lambda=1.55 \mu \mathrm{m}$ showed that a complete band gap can be achieved for triangular lattice with circular and hexagonal air holes in GaAs and for honeycomb lattice of circular GaAs rods.
\end{abstract}

Keywords: Photonic crystal; square lattice; triangular lattice; honeycomb lattice; plane wave expansion; mode field distribution; GaAs; dielectric contrast; filling fraction; gap maps

\section{INTRODUCTION}

Photonic crystals are periodic dielectric structures employed in a systematic way to achieve a band gap in a desired wavelength in applications of optical and electronic devices. These periodic structures are artificially arranged with the periodicity in micrometer to millimetre scales. In analogy to electrons in a semiconductor crystal, in photonic band gap materials, electromagnetic waves (EM) propagate in a structure with a periodically modulated dielectric constant. The periodic refractive index leads to the formation of photonic band gap not allowing any photons with a frequency in the range of the band gap to propagate. These artificial structures can have unlimited forms. Photonic band gap materials have already provided the possibilities of creating various novel applications with operating frequencies ranging from the microwave to the optical regime

Despite the early studies by Lord Rayleigh [1] in 1887 and Bykov [2] in 1972 on one dimensional photonic crystals, an extensive interest in these materials became established only after the independent studies of Yablonovitch [3] and John [4], concentrating on engineering possibility of higher dimensional structured materials exhibiting range of frequencies at which the propagating of EM waves is not allowed. Using plane wave expansion method Ho et al. [5] predicted the existence of complete band gap for a diamond lattice of spheres and established its dependence on dielectric contrast and filling fraction. By means of iterative optimization of approximate initial solutions through a parallel computing 
approach via block matrix diagonalization, Johnson and Joannopoulos [6] reduced the computational difficulty. The plane wave expansion method was adopted by Skoda [7] to compute the diffraction of two dimensional periodic band gap materials with finite thickness. Labilloy et al. [8] and Benisty et al. [9] used this method to predict the diffraction of triangular and square lattices of air holes in plane wave guides. Using analytical methods, an algorithm for computation of the spectrum and eigenmodes for two dimensional photonic crystals of square air rods was developed by Philal and Maradudin [10]. Villenuve and Piché [11] investigated the square lattice with both square and circular rods.

Due to fabrication difficulties two dimensional photonic crystals are more favoured than three dimensional photonic crystals. The plane wave expansion method applicable to any type of non-dispersive dielectric functions was implemented in the present work in modelling and simulating the band structures of two dimensional photonic crystals with square, triangular and honeycomb lattices. For the periodic dielectric function, the magnetic field vector was expanded using Bloch theorem leading to eigenvalue equation in matrix form. Standard eigenvalue equation was solved for different two dimensional lattice geometries for dielectric rods in air and air holes in dielectric mediums with circular, square and hexagonal cross sectional shapes. The effects of the parameters on the band gaps were studied and mode field distributions were calculated. The largest band gap generated was calculated by taking over all the filling fractions. The conventional gap maps were obtained by varying the radius or the width against lattice constant for both dielectric rods and air holes with different cross sections. The geometrical gap maps which give important statistical information in developing photonic crystal based devices were also calculated for telecommunication wavelength. Only the important diagrams are presented.

\section{PLANE WAVE EXPANSION}

Since the propagation of light in a photonic crystal is governed by Maxwell equations, the propagation of EM waves in a macroscopic, homogeneous, isotropic dielectric material with real dielectric constant $\varepsilon(r)$ with no dispersion was considered, restricting the conditions to space free of charge sources. Magnetic permeability $\mu(r)$ was assumed to be a constant. In a periodic dielectric structure, the Maxwell's equations for the electromagnetic waves can be written in terms of electric field $E$ and magnetic field strength $\boldsymbol{H}$ with angular frequency $\omega$ as,

$$
\begin{array}{ll}
\nabla \cdot \boldsymbol{H}(r)=0 & \boldsymbol{\nabla} \times \boldsymbol{E}(r)+\frac{i \omega}{c} \boldsymbol{H}(r)=0 \\
\nabla \cdot \varepsilon(r) \boldsymbol{E}(r)=0 & \boldsymbol{\nabla} \times \boldsymbol{H}(r)-\frac{i \omega}{c} \varepsilon(r) \boldsymbol{E}(r)=0
\end{array}
$$

These can be further simplified to [2],

$$
\nabla \times \frac{1}{\varepsilon(r)} \nabla \times \boldsymbol{H}=\frac{\omega^{2}}{c^{2}} \boldsymbol{H}(r) .
$$

Since $\varepsilon(r)$ is periodic, magnetic strength $\boldsymbol{H}$ can be expanded using the Bloch theorem: 


$$
\boldsymbol{H}(r)=\sum_{\boldsymbol{G}_{i}, \lambda} h\left(\boldsymbol{G}_{i}, \lambda\right) \hat{\boldsymbol{e}}_{\lambda} e^{i\left(\boldsymbol{k}+\boldsymbol{G}_{i}\right) \cdot \boldsymbol{r}} \quad\left(\boldsymbol{G}_{i}=h_{1} \boldsymbol{b}_{1}+h_{2} \boldsymbol{b}_{2}+h_{3} \boldsymbol{b}_{3}\right)
$$

where $\boldsymbol{G}_{i}$ are the reciprocal lattice vectors, $\boldsymbol{b}_{1}, \boldsymbol{b}_{2}, \boldsymbol{b}_{3}$ are the basis vectors in the reciprocal space, $h_{1}, h_{2}, h_{3}$ are integers and $\hat{\boldsymbol{e}}_{\lambda}$ are the two orthogonal unit vectors, both perpendicular to $\boldsymbol{k}+\boldsymbol{G}_{i}, \lambda$ representing the indices 1 and 2 . Substituting equation 3 into 2 and the matrix equation,

$$
\sum_{G^{\prime}}\left|\boldsymbol{k}+\boldsymbol{G} \| \boldsymbol{k}+\boldsymbol{G}^{\prime}\right| \varepsilon^{-1}\left(\boldsymbol{G}-\boldsymbol{G}^{\prime}\right)\left[\begin{array}{cc}
\hat{\boldsymbol{e}}_{2} \cdot \hat{\boldsymbol{e}}_{2}^{\prime} & -\hat{\boldsymbol{e}}_{2} \hat{\boldsymbol{e}}_{1}^{\prime} \\
-\hat{\boldsymbol{e}}_{1} \cdot \hat{\boldsymbol{e}}_{2}^{\prime} & \hat{\boldsymbol{e}}_{1} \cdot \hat{\boldsymbol{e}}_{1}^{\prime}
\end{array}\right]\left[\begin{array}{l}
h_{1}^{\prime} \\
h_{2}^{\prime}
\end{array}\right]=\frac{\omega^{2}}{c^{2}}\left[\begin{array}{l}
h_{1} \\
h_{2}
\end{array}\right]
$$

can be obtained. For 2D lattices, the material is homogeneous in $\mathrm{z}$ direction while periodic along $x$ and $y$ directions. The mirror symmetry along the $\mathrm{z}$ axis allows to classify the modes by separating them into two distinct polarization, the transverse-electric (TE) mode and the transverse-magnetic (TM) mode. Therefore equation 4 decoupled in to two equations which makes two unit vector sets unnecessary. The TM polarized mode and TE-polarized mode are given by equations 5 and 6 respectively

$$
\begin{aligned}
& \sum_{G^{\prime}}|k+G|\left|k+G^{\prime}\right| \varepsilon^{-1}\left(\vec{G}-\vec{G}^{\prime}\right) h\left(\overrightarrow{G^{\prime}}\right)=\frac{\omega^{2}}{c^{2}} h(\vec{G}) \\
& \sum_{G^{\prime}}(k+G) \cdot\left(k+G^{\prime}\right) \varepsilon^{-1}\left(\vec{G}-\overrightarrow{G^{\prime}}\right) h\left(\overrightarrow{G^{\prime}}\right)=\frac{\omega^{2}}{c^{2}} h(\vec{G}) .
\end{aligned}
$$

The absolute parts of these fields are used to calculate mode fields. $\varepsilon^{-1}\left(\boldsymbol{G}-\boldsymbol{G}^{\prime}\right)$ is the inverse of the Fourier transform matrix of $\varepsilon(r)$. In this standard eigenvalue problem, the difficulty in the analysis of the eigenvalue problem lies on Fourier transformation of the dielectric function. Performing a numerical fast Fourier transform is subjected to convergence problem and symmetry conditions become complex for some problems. The Fourier component of dielectric constant $\varepsilon(\boldsymbol{G})$ is,

$$
\varepsilon(\boldsymbol{G})=\frac{1}{A} \int_{A} \varepsilon(r) e^{-i \boldsymbol{G} \cdot r} d r
$$

where the integration is performed over the area $A$ of the one unit cell lattice. Periodic dielectric function in real space can be further simplified to,

$$
\begin{aligned}
\varepsilon(\boldsymbol{G}) & =f \varepsilon_{a}+(1-f) \varepsilon_{b} & & \text { for } \boldsymbol{G}=0 \\
& =\left(\varepsilon_{a}-\varepsilon_{b}\right) S(\boldsymbol{G}) & & \text { for } \boldsymbol{G} \neq 0
\end{aligned}
$$

where $f$ is the filling factor defined as the fraction of area occupied by the localized medium in one unit cell and $\varepsilon_{a}$ and $\varepsilon_{b}$ refer to the dielectric constants of the localized medium and the background respectively. The structure factor $S(\boldsymbol{G})$, depend only on the geometry of the localized medium and the lattice structure. This is given by, 
$S(\boldsymbol{G})=\frac{1}{A} \int_{A_{d}} e^{-i \boldsymbol{G} \cdot \boldsymbol{r}} d r$

The integration is carried over the area $A$ occupied by the localized medium in the unit cell. The structure factors for various shapes [12] are tabulated in Table 1.

Table 1. Structure factor for different shapes of rods.

\begin{tabular}{|c|c|}
\hline Shape & Structure factor \\
\hline $\begin{array}{c}\text { Circular } \\
\text { cylind- } \\
\text { rical } \\
\text { rod }\end{array}$ & $2 f \frac{J_{1}(G a)}{G a}$ (a-radius of the cylinder $)$ \\
\hline $\begin{array}{c}\text { Square } \\
\text { rod }\end{array}$ & $f \operatorname{sinc}\left(\frac{G_{x} a}{2}\right) \operatorname{sinc}\left(\frac{G_{y} a}{2}\right) \quad(a$ - side length of a square, $\operatorname{sinc} x=\sin x / x)$ \\
\hline $\begin{array}{c}\text { Hexa- } \\
\text { gonal } \\
\text { rod }\end{array}$ & $\frac{2 f}{3 G_{x} a}\left\{\sin \frac{a}{4}\left(3 G_{x}+\sqrt{3} G_{y}\right) \operatorname{sinc} \frac{a}{4}\left(G_{x}-\sqrt{3} G_{y}\right)+\sin \frac{a}{4}\left(3 G_{x}-\sqrt{3} G_{y}\right) \operatorname{sinc} \frac{a}{4}\left(G_{x}+\sqrt{3} G_{y}\right)\right\}$ \\
$(a-\operatorname{side}$ length of a hexagonal $)$
\end{tabular}

It is convenient to calculate for TM polarized mode, the displacement field $D$ and for TE polarized mode the magnetic field $H$ and these are given by

$$
\begin{aligned}
& D_{k}^{w}(r)=\frac{1}{|k|} \sum_{G}|k+G| h_{1}(G) \cdot e^{i(k+G) \cdot r} \hat{z} \\
& H_{k}^{w}(r)=\sum_{G} h_{1}(G) \hat{z} \cdot e^{i(k+G) \cdot r}
\end{aligned}
$$

The plane wave expansion method was used to simulate band structure of different photonic lattices formed from rods of different cross sectional shapes. For each photonic crystal structure, the possibility of TE, TM and complete band gap was studied carefully using band diagrams.

\section{SQUARE LATTICE}

For a two dimensional square photonic crystal formed by circular dielectric rods of radius $r$ with lattice constant $a$, the basis vectors and the first Brillouin zone are shown in Figure 1. The band structure and the density of states for a square lattice with basis lattice vectors $(1,0,0) a$ and $(0,1,0) a$ with the atom positioned at $(0,0,0) a$ formed from circular GaAs dielectric rods $(\varepsilon=13)$ of radius $r=0.16 a$ with filling fraction $f=\pi r^{2} / a^{2}=0.08$ and square GaAs dielectric rods of width $w=0.6 a$ with filling fraction $f=w^{2} / a^{2}=0.06$ in 
air are shown in Figure 2. The frequency is expressed as a dimensionless ratio $\omega a / 2 \pi c$ in the vertical axis and the parallel plane wave vector along the triangular edge of the irreducible Brillouin zone ГМХГ in the $\mathrm{x}$ axis. For the square lattice formed from circular GaAs dielectric rods, although a complete band gap was observed for TM mode in all direction between first and second band, there was no gap for the TE mode. For the square lattice formed from square GaAs dielectric rods, the TM band gaps are observed for three frequency ranges, and the density of states indicate that there are no modes in the band gap. The band gap observed between first and second bands can be useful in designing band stop filters and polarisers for lower frequencies. The yellow region at normalized high optical frequency range between 0.69-0.65 indicates a complete gap.
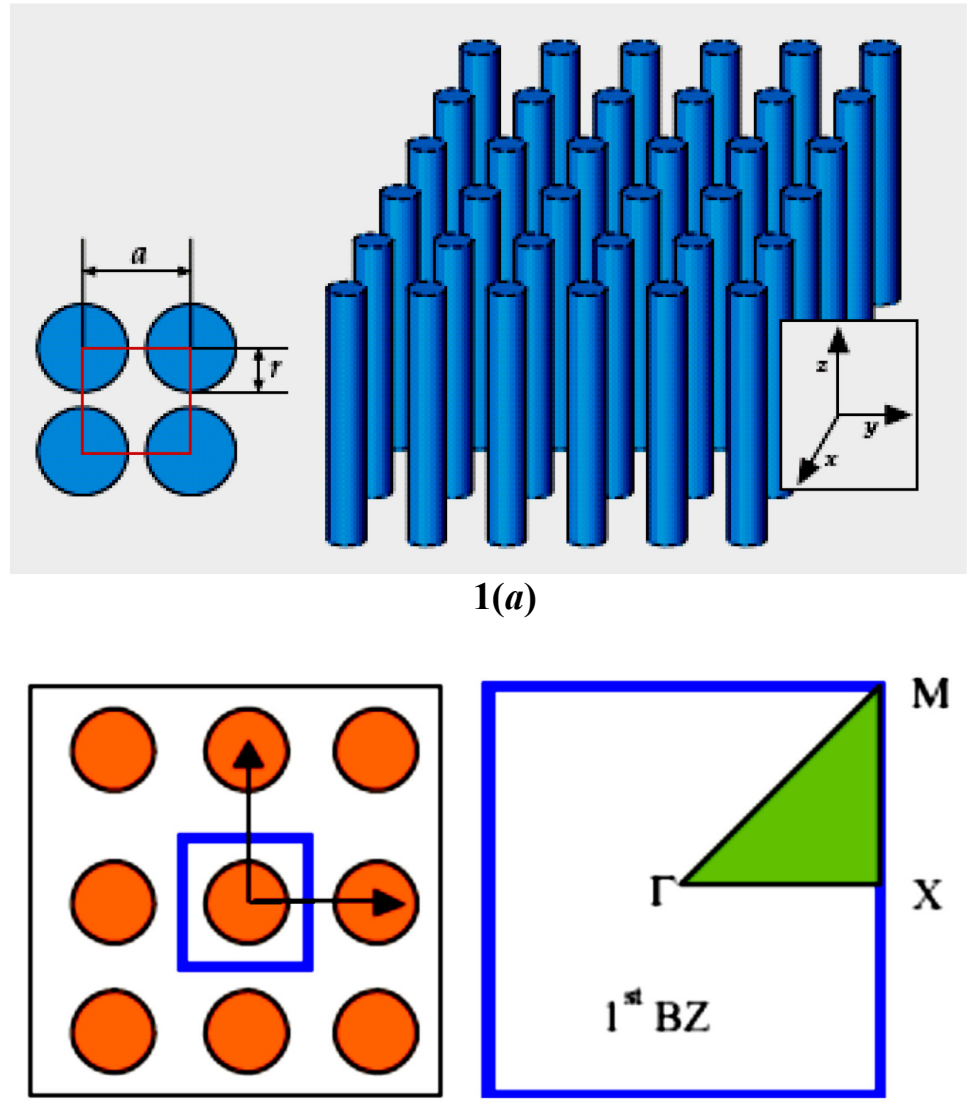

$1(b)$

Fig. 1. (a) Two dimensional square photonic crystal of circular rods of radius $r$ with lattice constant $a$. (b) The unit cell, basis lattice vectors and first Brillouin zone. 

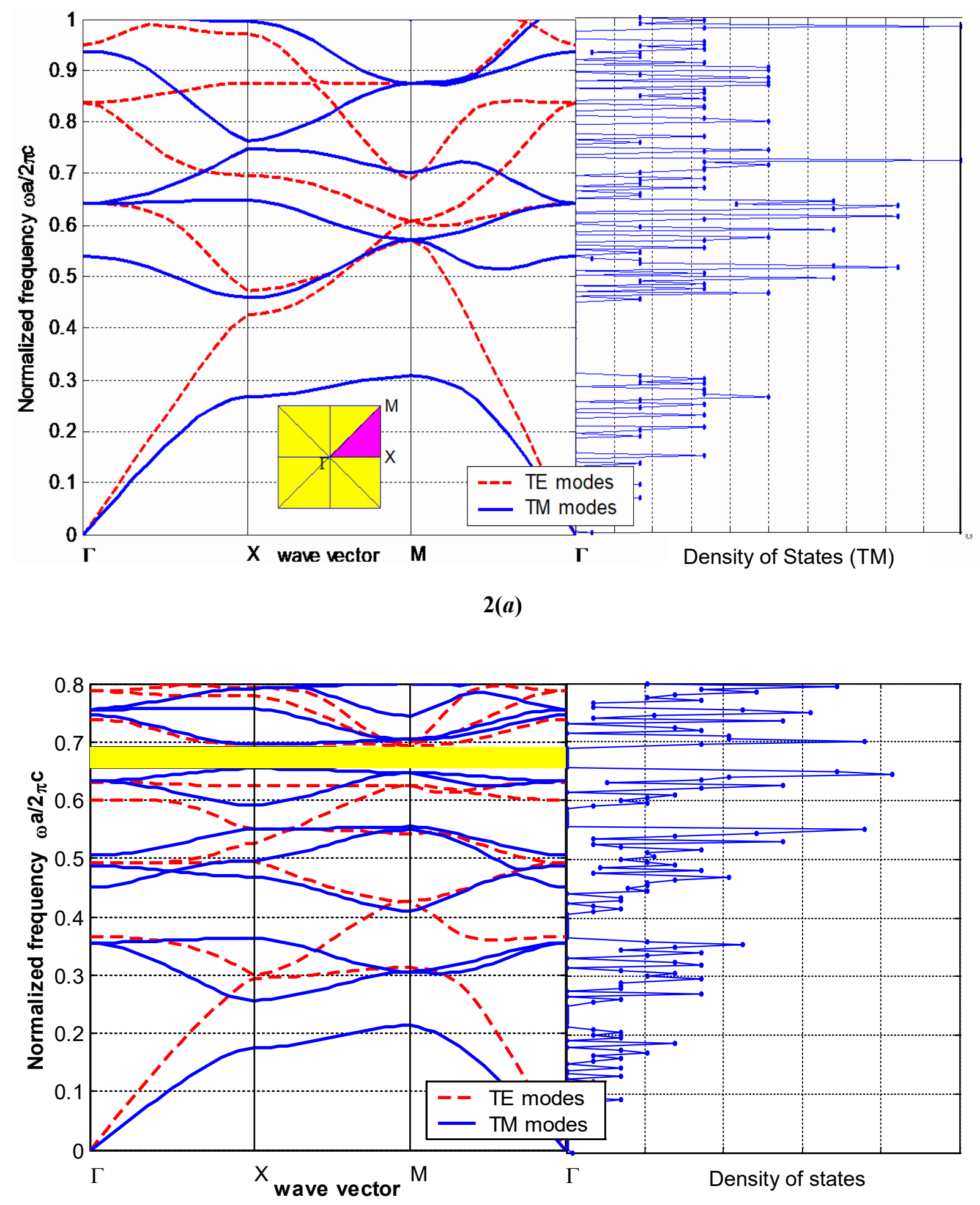

2(b)

Fig. 2. The photonic band structure $(2(a))$ and density of states $(2(b))$ for a square array for dielectric rods in air $\varepsilon_{a}=13$ and $\varepsilon_{b}=1$ (a) circular rods $(r=0.16 a, f=0.08)$, (b) square rods $(w=0.6 a, f=0.36)$. 


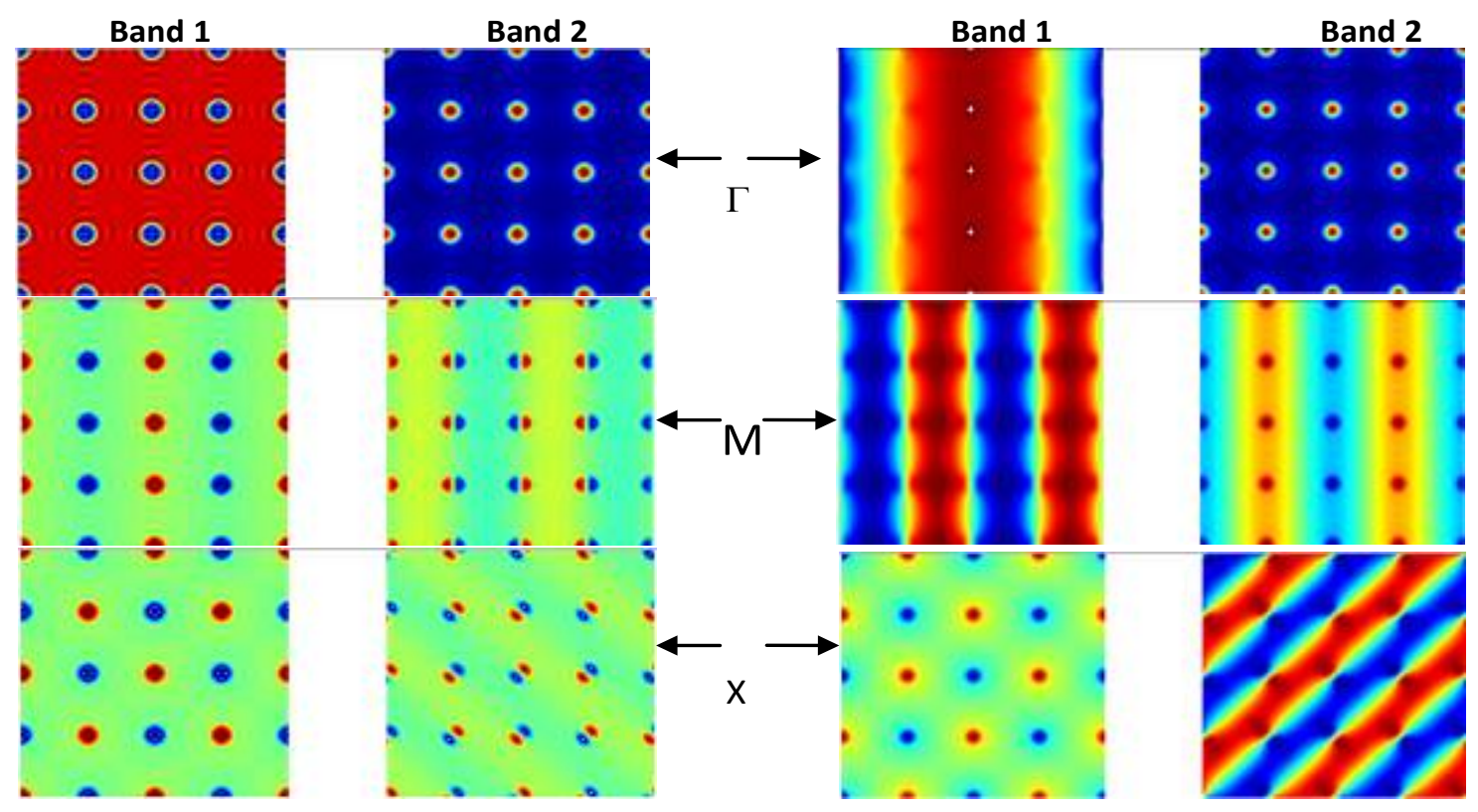

$D$ field for TM mode

$H$ field for TE mode

3(a)

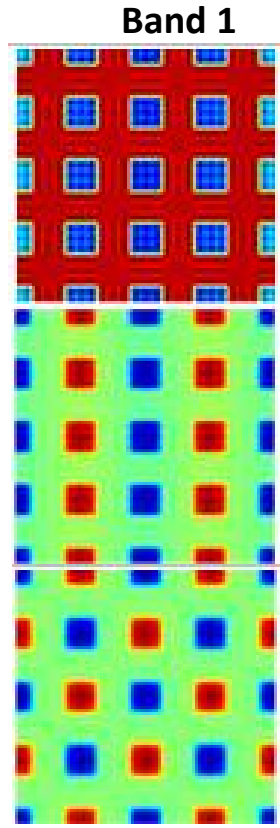

$D$ field for TM mode
Band 2

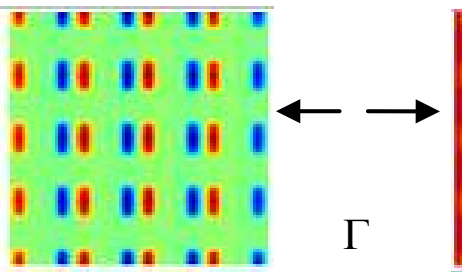

11111111

$111111114 \rightarrow$

| 111111 |

$\because \because \because \because:$

$10 \mathrm{~s}$

1,15

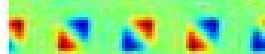

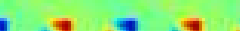

Band 1
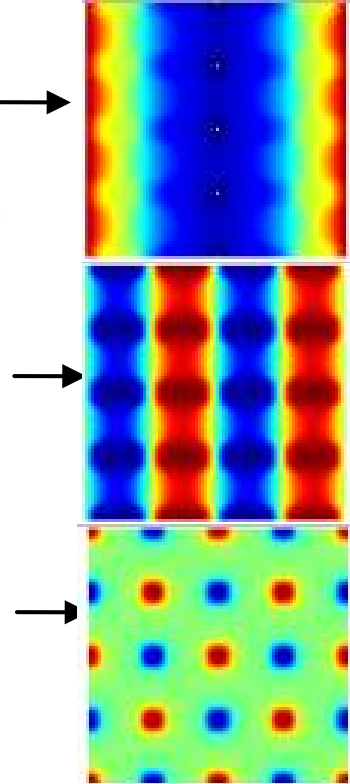

$H$ field for TE mode
Band 2

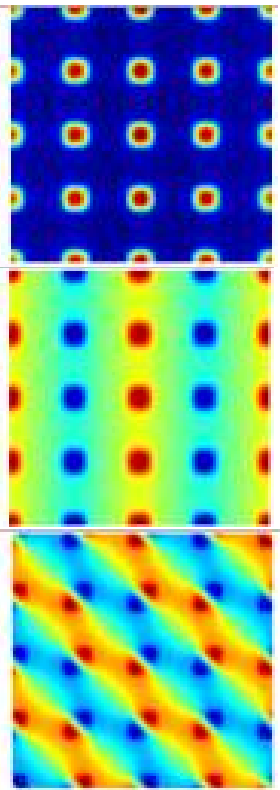


$\mathrm{X}(\pi / a)(1,0,0)$ and $\mathrm{M}(\pi / a)(1,1,0)$ points for circular and square GaAs rods in air for TM and TE modes and these are presented in the Figure 3(a) and 3(b) respectively. The dark red and blue represent the high $\varepsilon$ region. The magnitude of the intensity is denoted by the colour variation, red denoting a positive field and blue denoting a negative field. Displacement field at $\Gamma$-point is same for each unit cell. Since the X-point is at the edge of Brillouin zone, the fields alternate in each unit cell in the direction of the $k_{z}$ wave vector, making the wave fronts parallel to $y$-axis. At $\mathrm{M}$ point, phases of the neighbouring cells alternate with each other resulting a check-board pattern, similar to a plane wave propagating in the $x+y$ direction. The displacement field patterns for band 1 (dielectric band) shows a high energy concentration inside the high $\varepsilon$ region and the field pattern of band 2 (air band) shows that some energy ofhigh- $\varepsilon$ regions is expelled into lower- $\varepsilon$ region. For circular rods, at $\mathrm{X}$ and $\mathrm{M}$ points, the first mode is a monopole and the second mode is a dipole whereas at $\Gamma$-point both modes are monopoles. For square rods, at $\mathrm{X}, \Gamma$ and $\mathrm{M}$ points, the first mode is a monopole and second mode is a dipole leading to a TM band gap between band 1 and band 2 .

Since circular and square dielectric rods in air give rise to large TM band gaps, the variation of this gap width $\Delta \omega$ to mid gap frequency $\omega_{m}$ ratio with dielectric contrast and filling factor were calculated and these are presented in Figure 4. The largest band gap generated was calculated by taking over all the filling fractions. Minimum dielectric index contrast of 3 was enough to open a TM band gap for both square and circular rods. The largest band gap width for a dielectric contrast increased gradually and tended to a constant. A large band gap width, about $40 \%$ gap width to mid gap ratio was observed for GaAs rods. The circular and square rods behaved in a similar way for TM mode, the highest gap occurring for a filling factor of 0.1 with corresponding radius $0.178 a$ and width of $0.316 a$ respectively. In order to have a large TM gap, dielectric rods of smaller radius or width must be used.

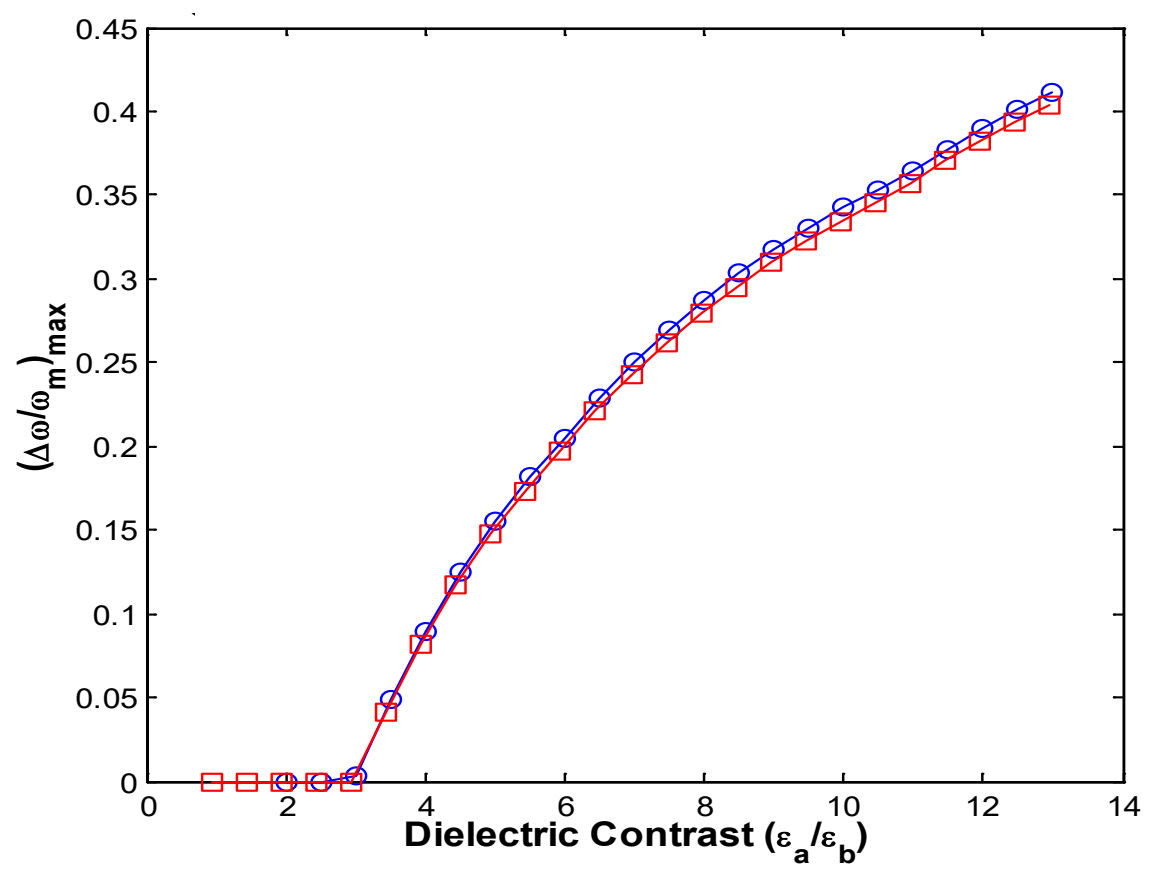

4(a) 


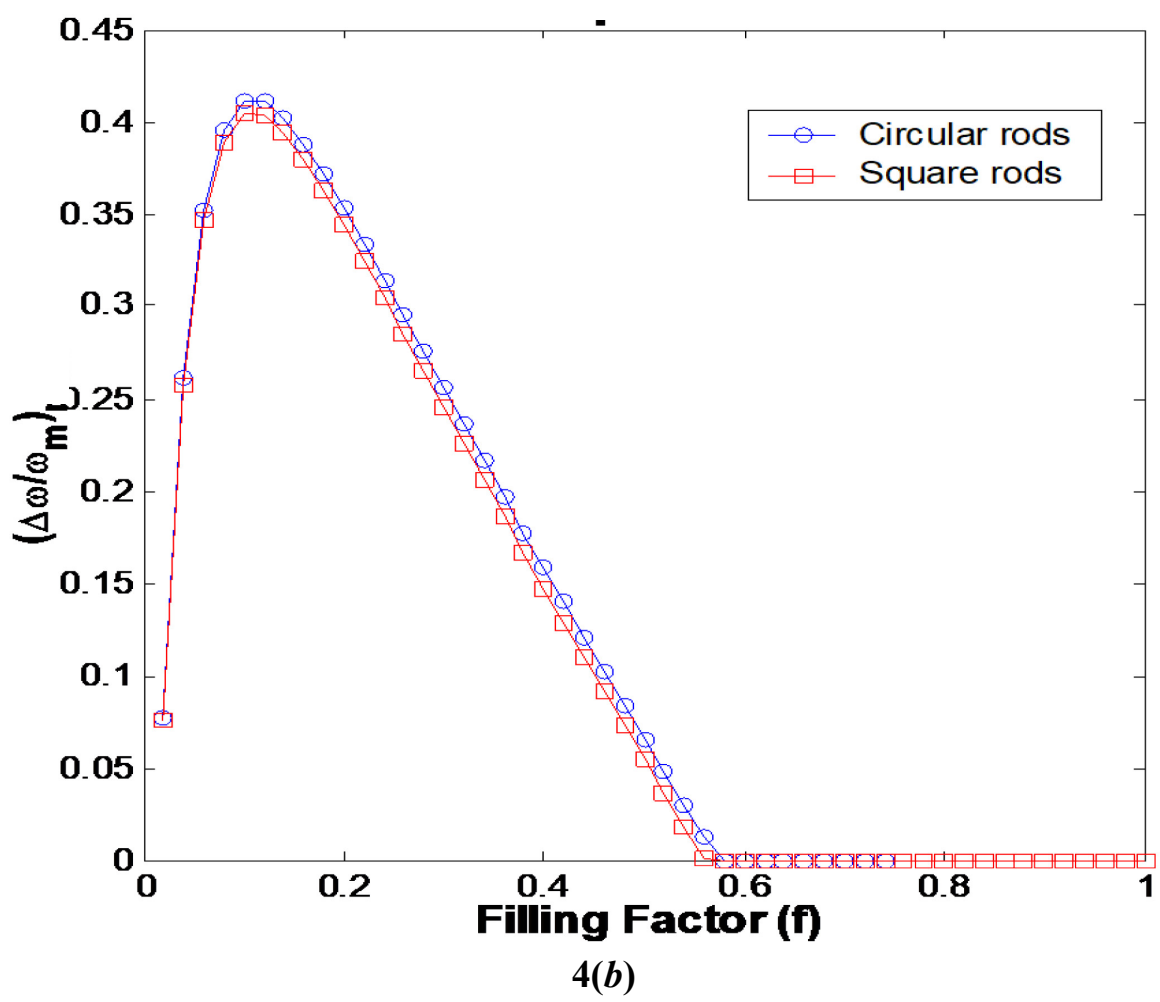

Fig. 4. for square lattice of circular and square GaAs rods in air (a) maximum TM band gap width to mid gap ratio with dielectric contrast and $(b)$ TM band gap width to mid gap ratio with filling factor.

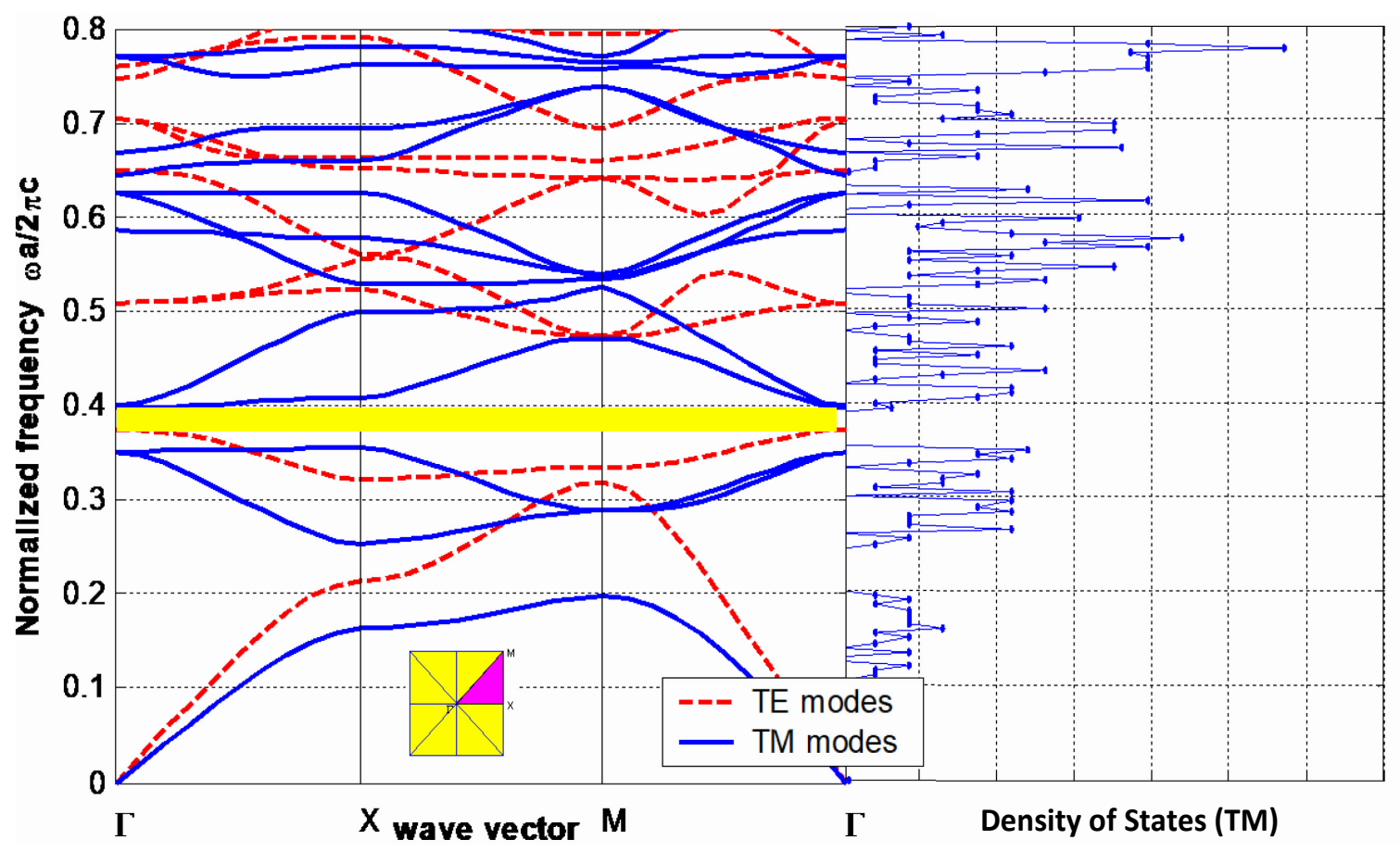

5(a) 


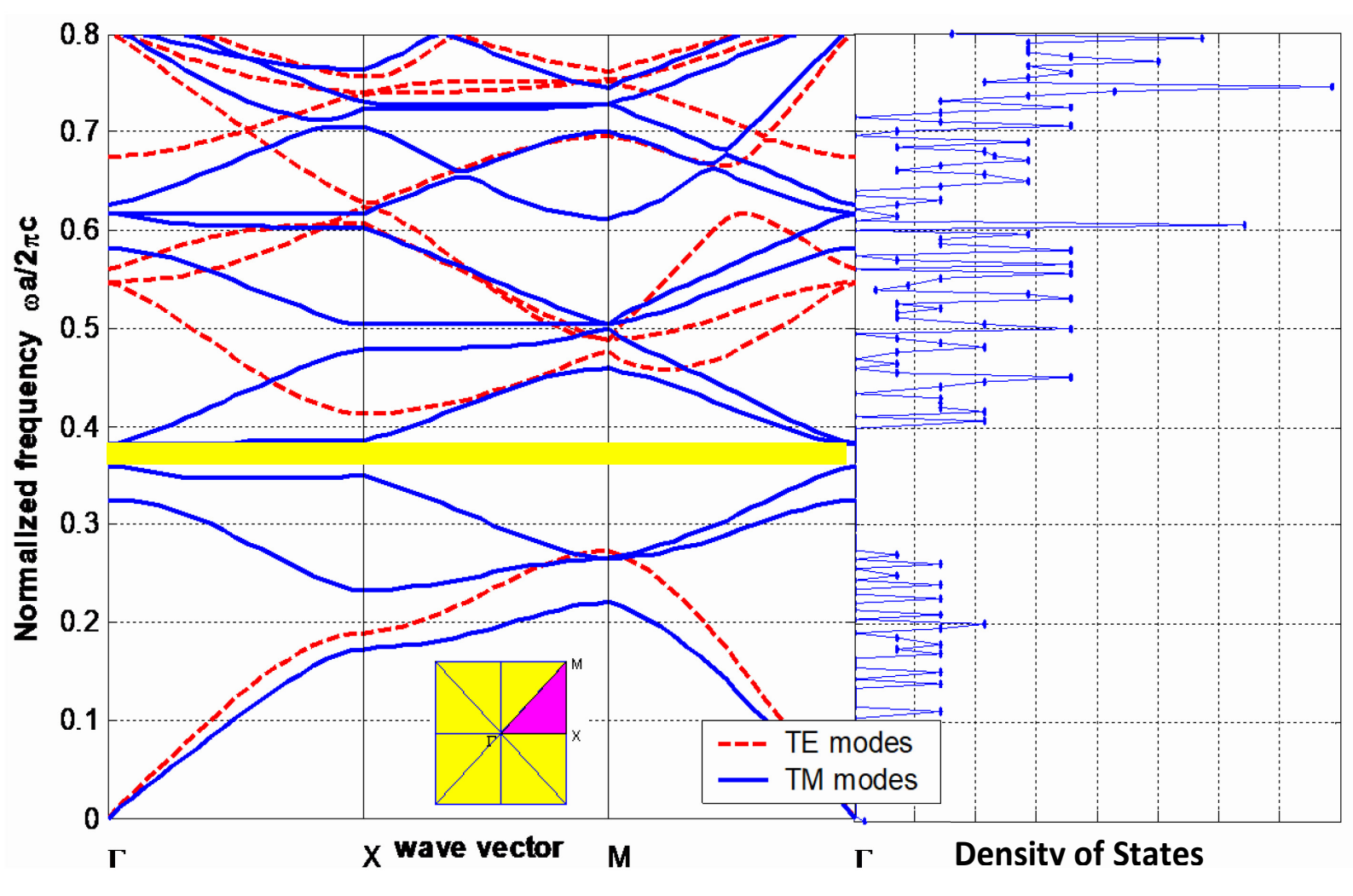

$5(b)$

Fig. 5. Band structures of two dimensional square photonic crystals of $(a)$ circular air columns of radius $r=0.48 a$ and $(b)$ square air holes of width $w=0.85 a$ in a medium $\varepsilon_{a}=1, \varepsilon_{b}=18, f=0.72$.

By increasing the dielectric contrast, complete band gaps for both polarizations can be obtained for air holes drilled in dielectric mediums. The band diagram obtained for a square lattice with circular air holes radius $0.48 a$ and square air holes with width $0.85 a$ drilled in a dielectric medium $\varepsilon=18$ is shown in Figure 5. For circular holes the TM band gap between third and fourth bands and TE band gap between second and third bands overlap at around 0.386 , giving rise to a complete band gap width of $0.023(\omega a / 2 \pi c)$.

This gap is useful in fabricating a perfect dielectric mirror from a photonic crystal. For square holes a complete band gap for both polarizations was observed between normalized frequencies 0.36 and 0.38. $D$ fields of TM modes for band 3 and band 4 and $H$ fields of TE modes for band 1 and band 2 for square air holes of width $w=0.85 a$ drilled in a dielectric medium with $\varepsilon=18$ is presented in Figure 6. The mode field distribution patterns for the structure concentrate most of the energy in the high $\varepsilon$ region. Slight difference in the displacement field patterns for the modes opens the TM band gap for high dielectric contrast.

The complete gap width to mid gap frequency ratio with dielectric contrast and filling factor for circular and square air holes in a dielectric medium is presented in Figure 7. The array of circular holes requires a significant lower dielectric contrast to generate a band gap than an array of square holes. Minimum index contrast needed to open a photonic gap for circular rods was 7.5 whereas for square holes it was 13. The lattice consisting of circular holes has a large gap to mid gap ratio when the dielectric contrast is below 16.3. 


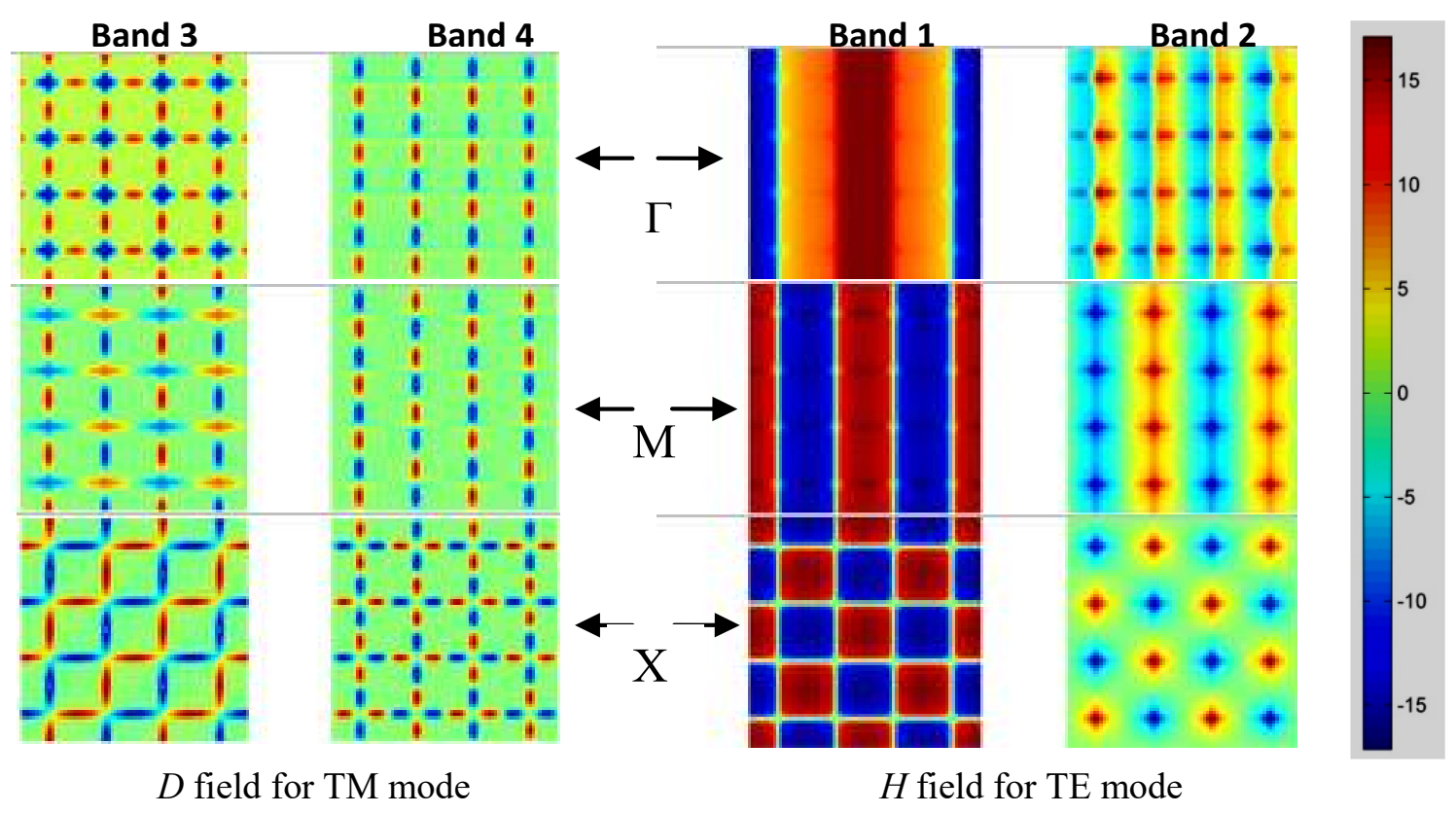

Fig. 6. Mode field distribution of square lattice of square air holes $\varepsilon_{\mathrm{a}}=1$ and $\varepsilon_{\mathrm{b}}=18$ with $w=0.85 \mathrm{a}$. $D$ fields of TM modes for band 3 and band 4 and $H$ field of TE modes for band 1 and band 2 at $\Gamma$ point (top), M point (middle) and X point (bottom).

The variation of the complete gap width with filling factor is presented Figure $7(b)$ for the background material with a dielectric constant 18. The overlap of TM and TE waves could be achieved for smaller filling fractions in square rods than circular rods. For this particular dielectric contrast, array of square rods yields, a larger gap to mid gap ratio about 9 $\%$. The discontinuities of the figure indicate the change in the upper and lower bands of the overlap TM and TE gaps.

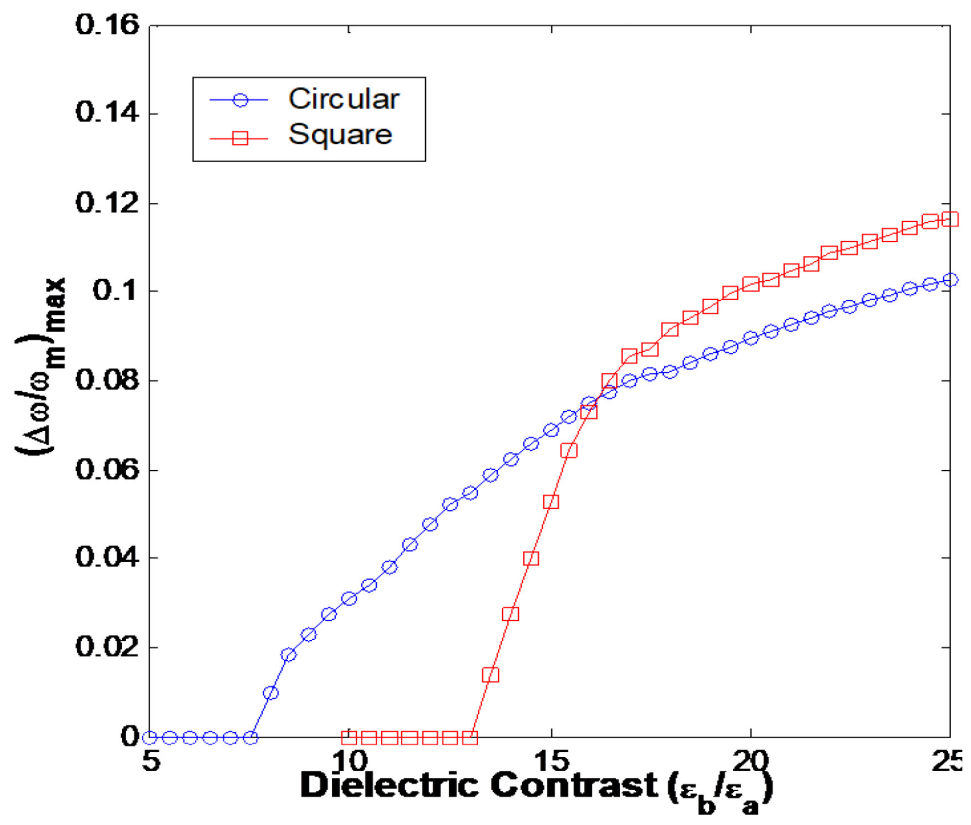

7(a) 


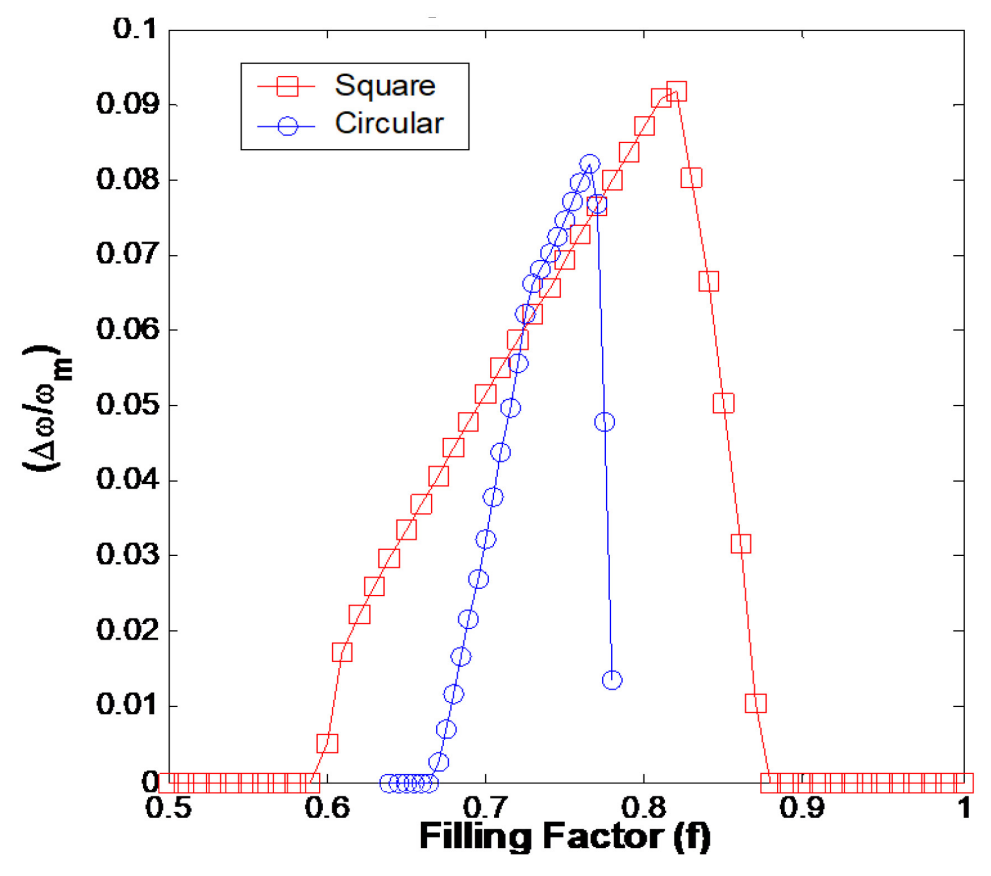

$7(b)$

Fig. 7. (a) Maximum complete band gap width to mid gap ratio with dielectric contrast and $(b)$ complete band gap width to mid gap ratio with filling factor for square lattice of air holes with

$$
\varepsilon_{\mathrm{a}}=1, \varepsilon_{\mathrm{b}}=18 .
$$

\section{TRIANGULAR LATTICE}

Two dimensional triangular photonic crystal with a lattice constant $a$, formed by circular air columns of radius $r$ in a dielectric medium is shown in Figure 8 [12]. The basis lattice vectors are $(1,0,0) a$ and $(1 / 2, \sqrt{3} / 2,0) a$ with atom positioned at $(0,0,0) a$. The band structure obtained for circular air holes of radius $r=0.48 a$ drilled in GaAs presented in figure $9(a)$, indicate a large complete band gap between 0.403-0.512( $\omega \mathrm{a} / 2 \pi \mathrm{c})$ with a mid gap at $0.476(\omega a / 2 \pi c)$. Gap to mid gap ratio is $19.15 \%$. If the mid band wavelength is chosen as the telecommunication wavelength $\lambda=1.55 \mu \mathrm{m}$, then the value of the lattice constant, filling fraction and the radius of the rod must have the values $a=0.7373 \mu m, f=0.84$, $r=0.4096 \mu m$ respectively.

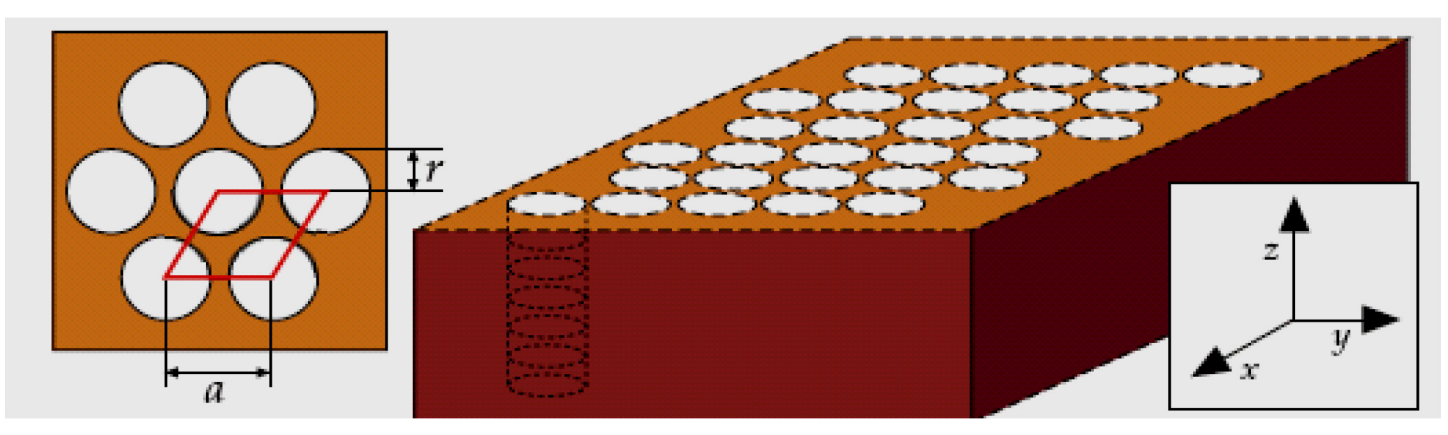



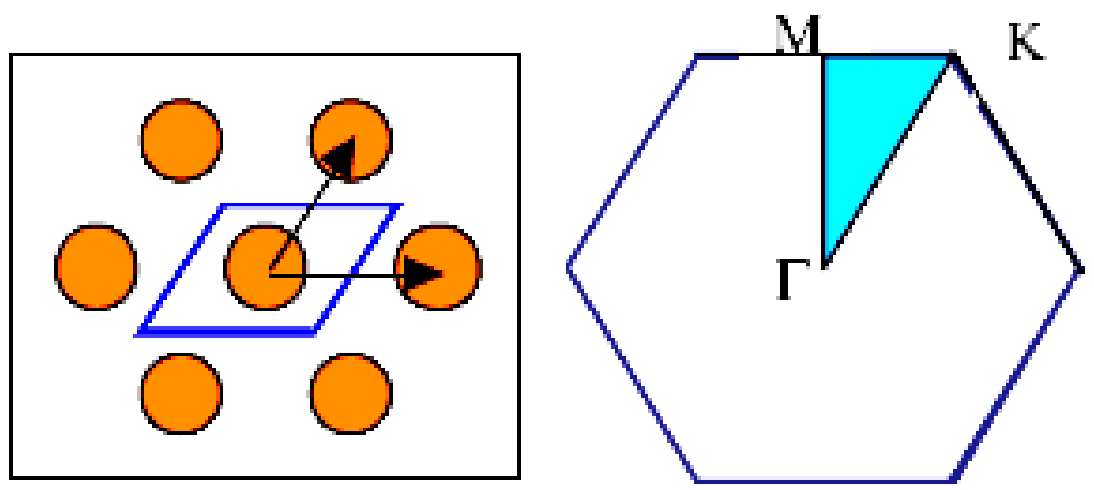

B

Fig. 8. A: (Right) A two dimensional photonic crystal of air columns of radius $r$ in dielectric substrate. (Left) Triangular lattice with a lattice constant $a$. B: (Right) Briliouin zone. (Left) Unit cell in blue and basis lattice vectors.

Triangular lattice can be also formed from hexagonal cross sectional air holes having the same physical shape as the lattice. For hexagonal air holes with width size $w=0.48 a$ drilled in GaAs, although a complete band gap was observed, the band gap width was smaller than that of circular air holes (Figure $9(b)$ ). Gap mid ratio was $8 \%$.

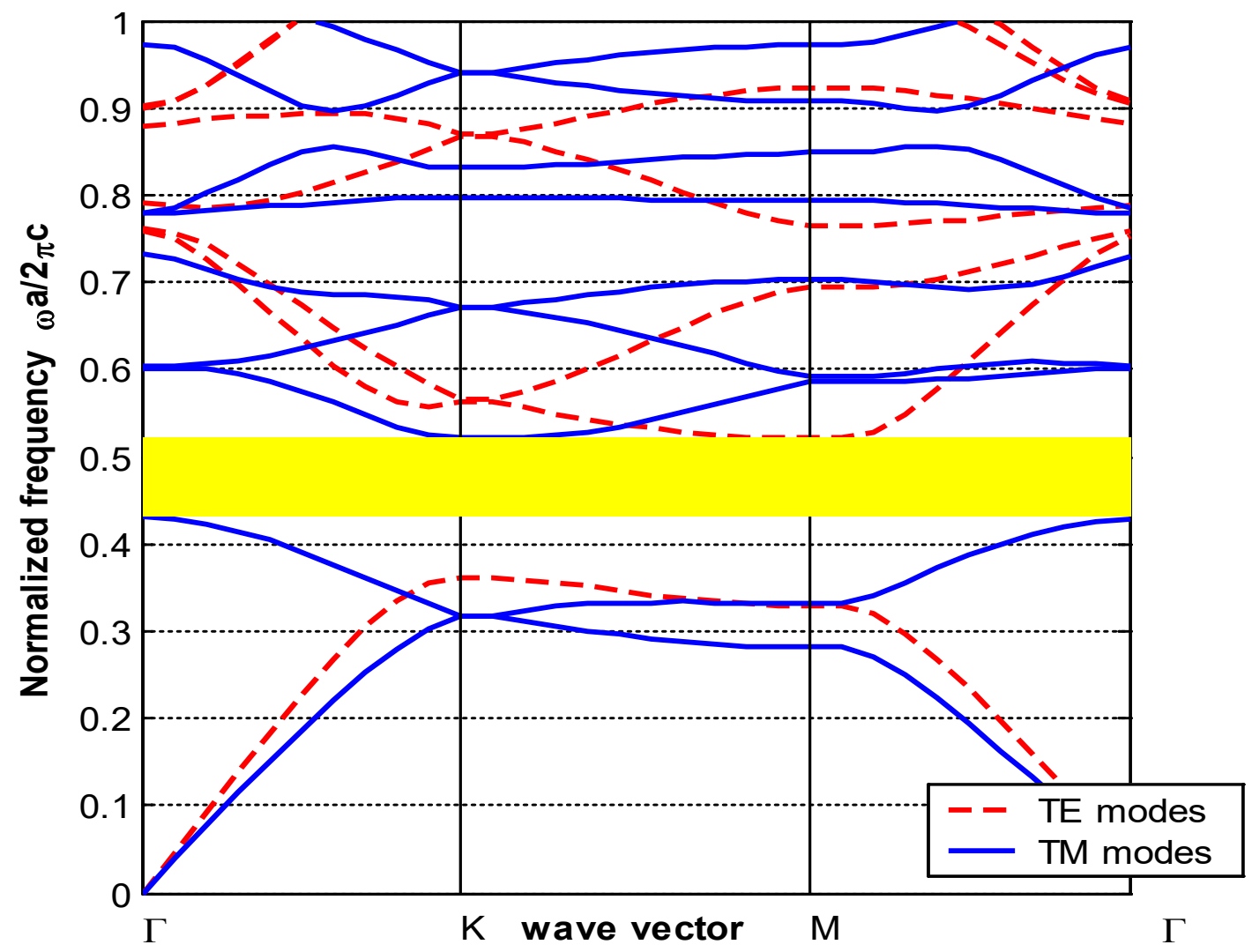

9(a) 


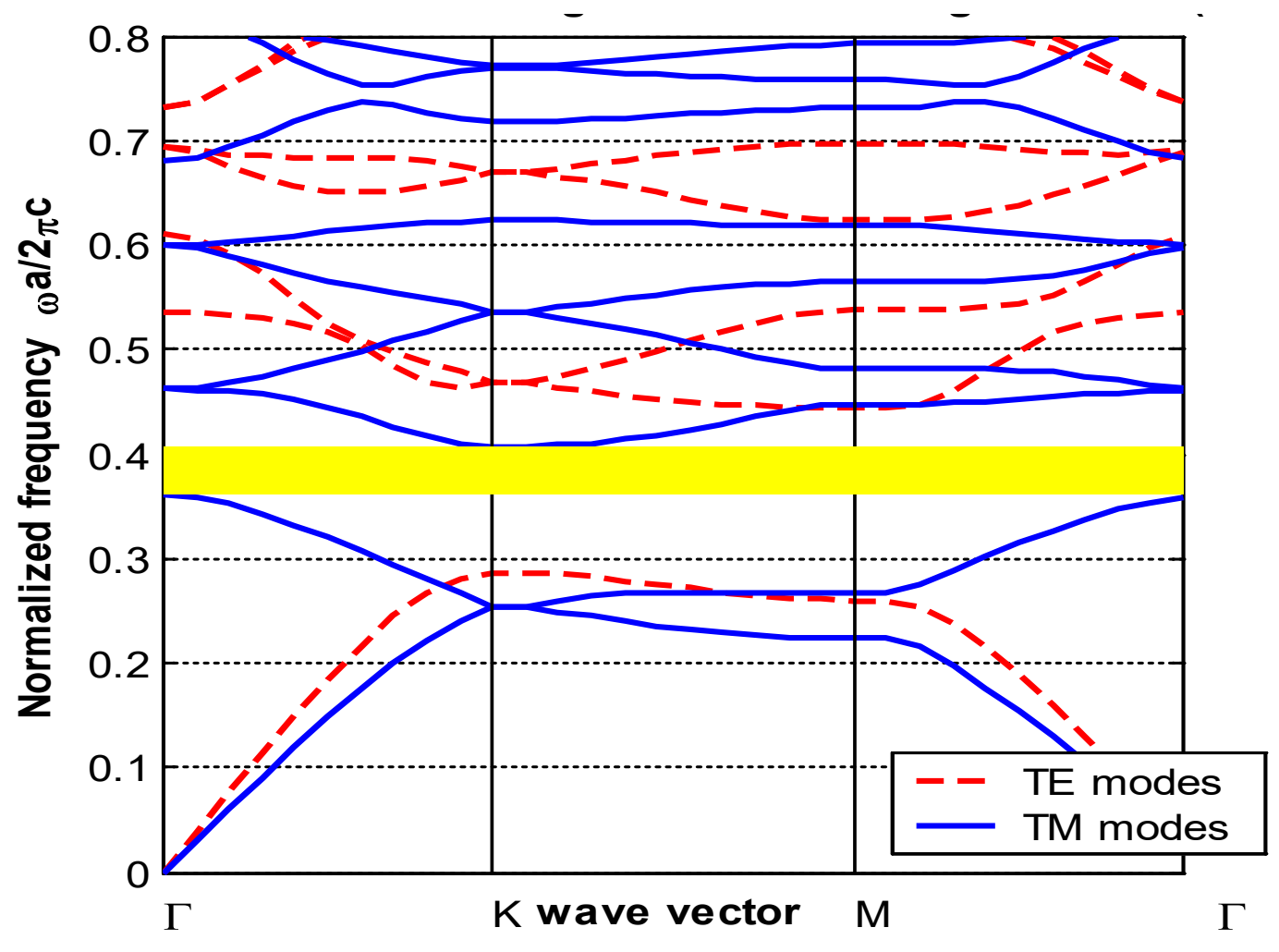

9(b)

Fig. 9. Band structure for two dimensional triangular photonic crystals of $(a)$ circular air holes of radius $r=0.48 a, f=0.84$ and $(b)$ hexagonal air holes with $w=0.48 a, f=0.69$ in GaAs.

For two dimensional triangular photonic crystals formed from circular and hexagonal air holes drilled in GaAs, the mode field distributions were calculated for points at the upper and lower frequencies of the corresponding bands. Figure 10 shows the mode field distribution pattern of circular air holes drilled in GaAs for displacement field for TM modes at $\Gamma$ point of band 2 and $\mathrm{K}$ point of band 3 as well as magnetic field distribution for TE modes at band 1 and 2 at $\mathrm{K}$ point and band 2 at $\mathrm{M}$ point.

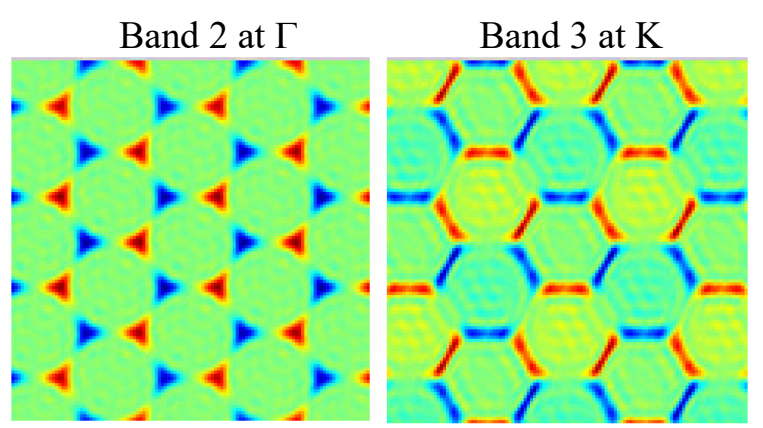

$D$ field for TM mode

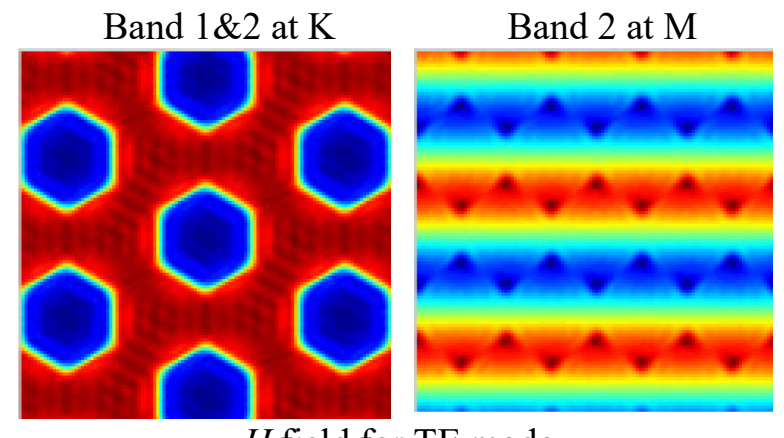

$H$ field for TE mode

Fig. 10. Mode field distribution of triangular lattice of circular air holes drilled in GaAs. $r=0.48 a$, $\varepsilon_{\mathrm{a}}=1$ and $\varepsilon_{\mathrm{b}}=13$. 

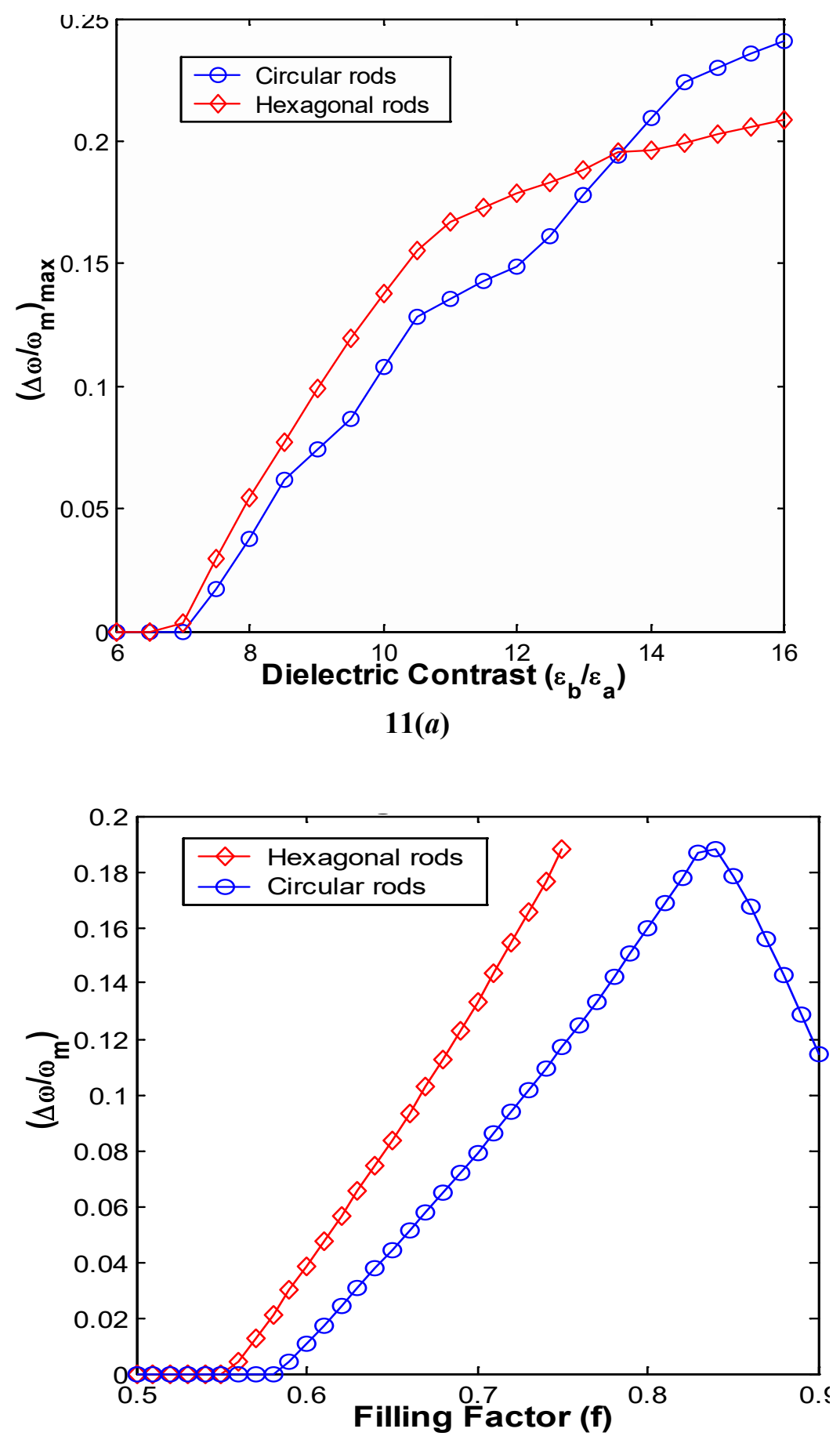

$11(b)$

Fig. 11. For triangular lattice of circular and hexagonal air holes, $(a)$ maximum complete band gap width to mid gap ratio with dielectric contrast and $(b)$ complete band gap width to mid gap ratio with filling factor for $\varepsilon_{a}=1, \varepsilon_{b}=13$.

The distribution shows a large difference, the modes at $\Gamma$ point of band 2 concentrating more energy in the 'spots' while the modes at $\mathrm{K}$ point of band 3 concentrating more energy in the 'veins'. The difference in magnetic field distributions between corresponding points, result a TE gap between the respective bands. The largest complete band gap generated in a 
triangular lattice with circular and hexagonal air holes with dielectric contrast are shown in Figure 11. According to the figures, both arrays of circular holes and hexagonal holes require lower dielectric contrast to generate a complete band gap. The minimum index contrast required for both circular and hexagonal air holes is 7 . The hexagonal holes have a large optimal gap to mid gap ratio for dielectric contrast below 13. The variation of the complete gap width with the filling factor is presented in Figure 11(b) for GaAs background material. A photonic gap can be obtained for smaller filling fractions for hexagonal holes than that for circular holes. For this particular dielectric contrast, both kind of arrays yielded nearly equal maximum gap width of about $18 \%$ gap ratio. For both two dimensional triangular photonic crystals formed by $\operatorname{circular}(r=0.2 a, f=0.15)$ and hexagonal $(w=0.2 a, f=0.12) \mathrm{GaAs}$ dielectric rods, although a TM gap width of $0.17(\omega a / 2 \pi c)$ was observed around mid gap frequency $0.35(\omega a / 2 \pi c)$, no TE gap was observed. The variation of gap width to mid gap frequency ratio with dielectric contrast for these crystals are shown in Figure 12.

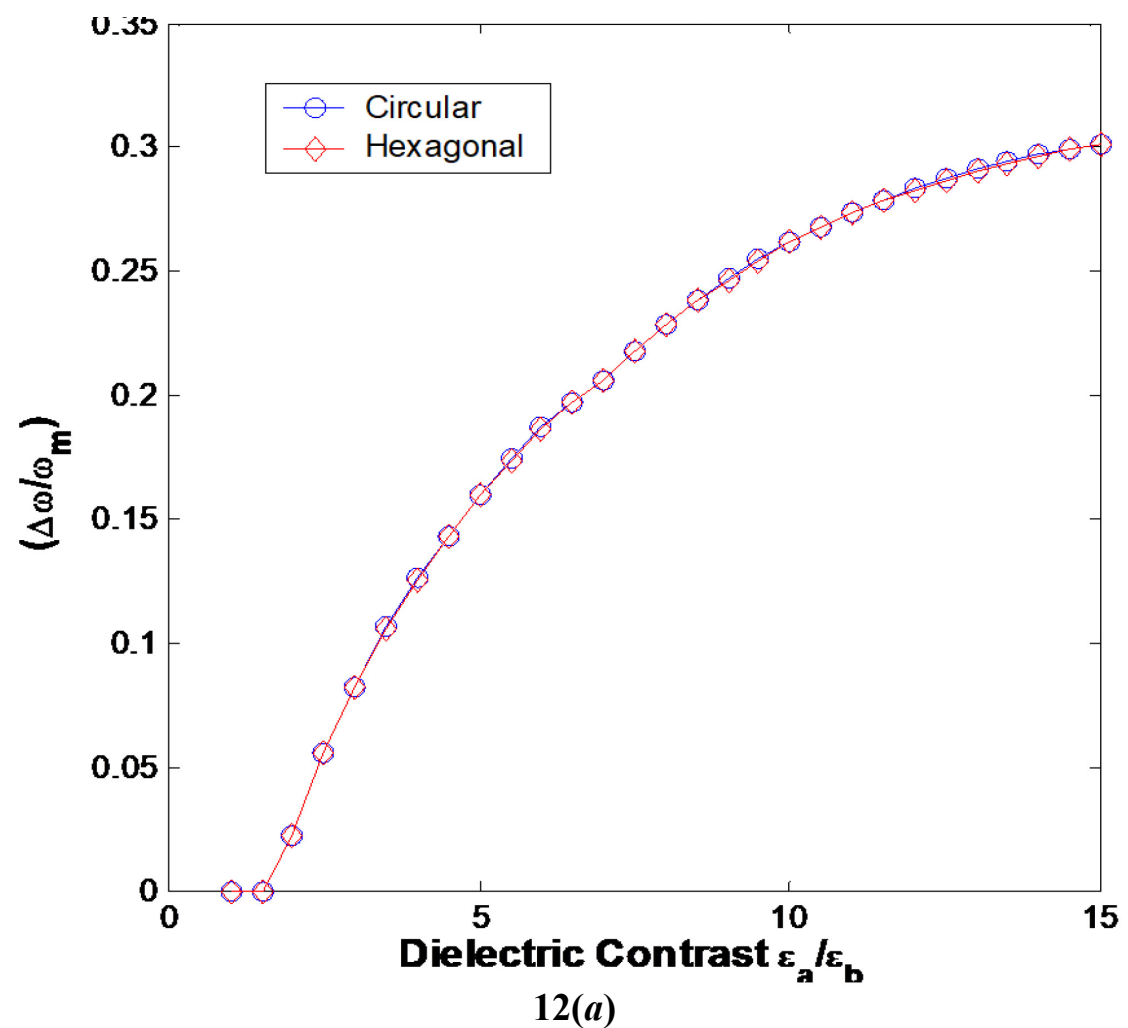

Both variations look exactly the same for the circular and hexagonal rods. The minimum index contrast to open a TM gap for both these rods occurs for the dielectric contrast of 2. The variation of gap width to mid gap frequency ratio with filling fraction for GaAs rods in air is presented in Figure 12(b). Since this is a disconnected lattice, the largest gap occurs for small filling fractions. The TM band gap for triangular lattice does not depend on the shape of the rods. Both variations look exactly the same for the circular and hexagonal rods. The minimum index contrast to open a TM gap for both these rods occurs for the dielectric contrast of 2 .

The variation of gap width to mid gap frequency ratio with filling fraction for GaAs rods in air is presented in Figure 12(b). Since this is a disconnected lattice, the largest gap occurs for small filling fractions. The TM band gap for triangular lattice does not depend on the shape of the rods. 


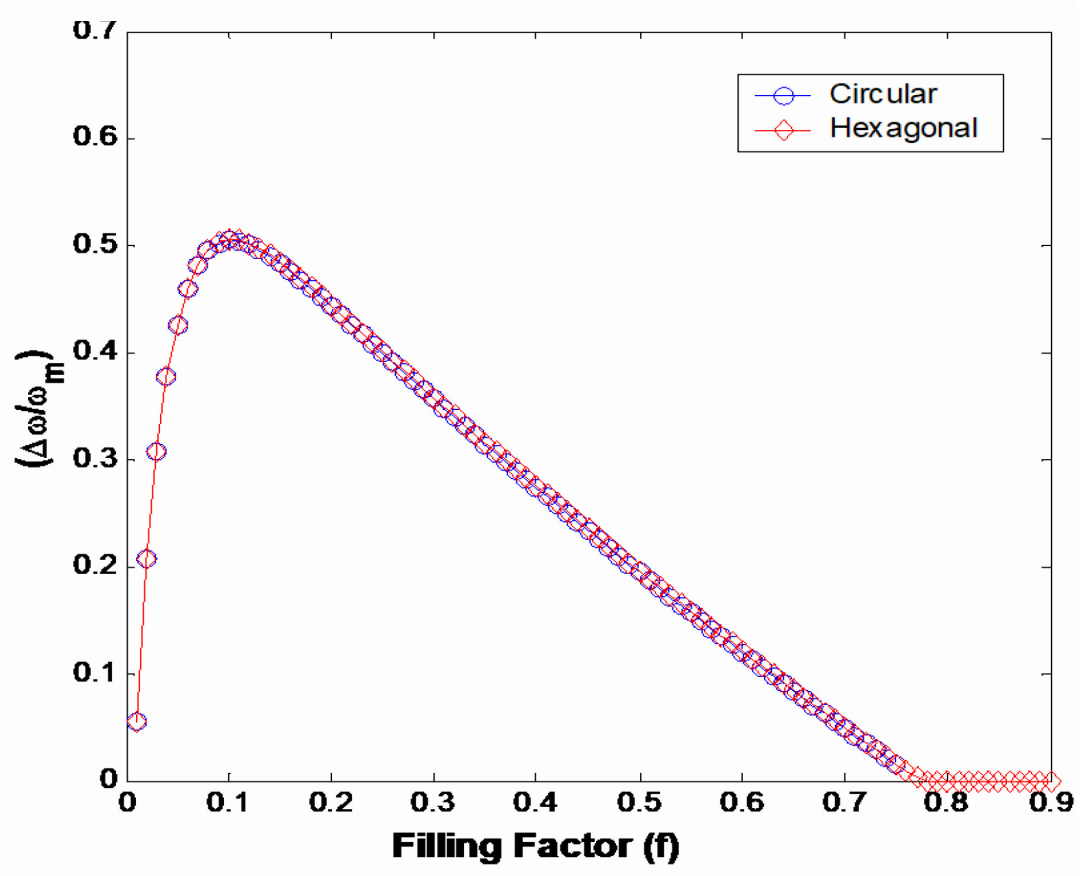

$12(b)$

Fig. 12. For triangular lattice of circular and hexagonal GaAs rods $(a)$ the maximum TM band gap width to mid gap ratio with dielectric contrast and $(b)$ TM band gap width to mid gap ratio with filling factor.

\section{HONEYCOMB PHOTONOIC LATTICE}

For the two dimensional honycomb lattice or a hexaganoal lattice, the unit cell, basis lattice vectors and Brillouin zone are shown in Figure 13.
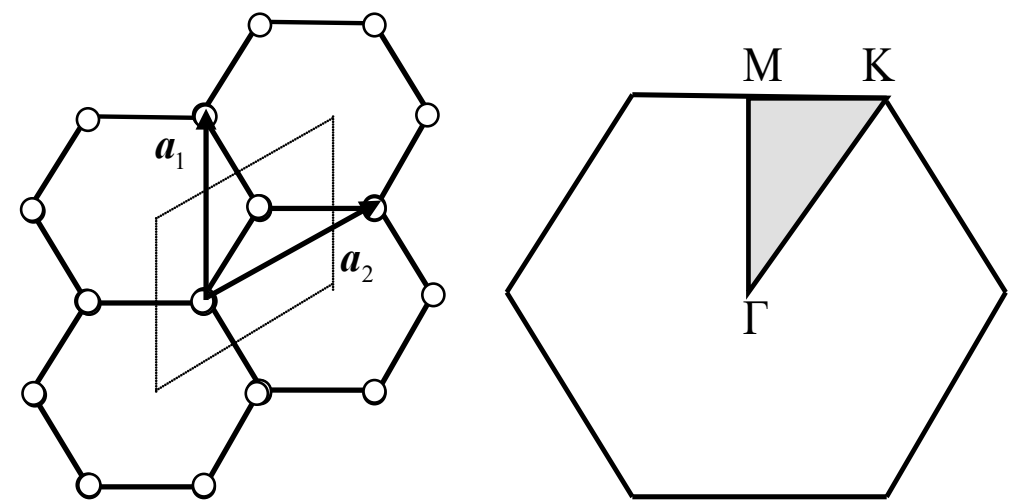

Fig. 13. 2D honycomb lattice. Left- unit cell and basis lattice vectors. Right- Brillouin zone. 

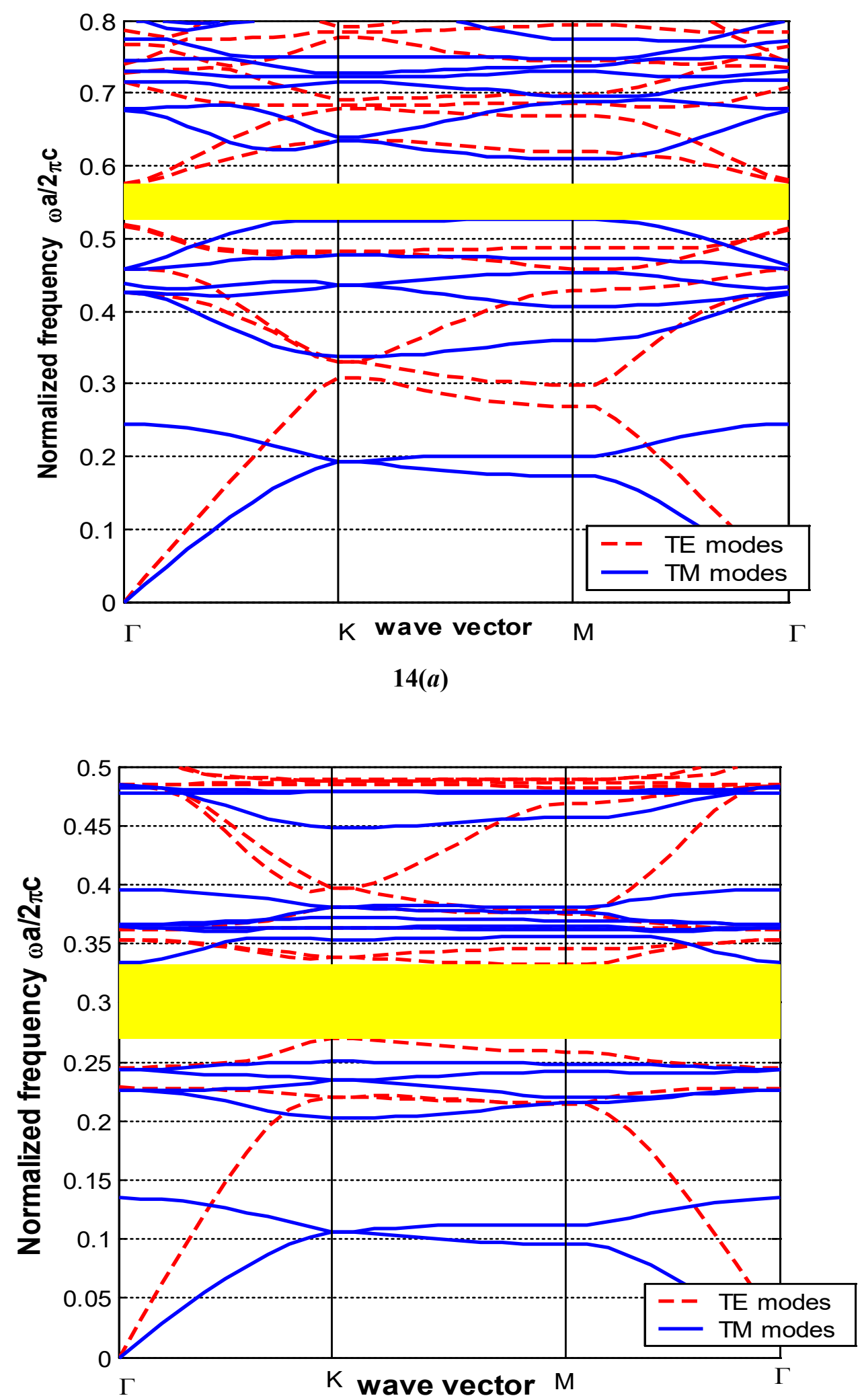

$14(b)$

Fig. 14. Band structure for two dimensional honycomb crystal of GaAs ( $a$ ) circular rods with $r=0.23 a, f=0.13$ and $(b)$ hexagonal rods with $w=0.3 a, f=0.54$ in air. 
The basis lattice vectors are $(1,0,0) a \sqrt{3}$ and $(1 / 2, \sqrt{3} / 2,0) a \sqrt{3}$ with atoms positioned at $(1 / 2, \sqrt{3} / 2,0) a$ and $(-1 / 2,-\sqrt{3} / 2,0) a$. The structure factor for honey comb lattice with hexagonal rods presented in Table 1 , must be multiplied by an additional term $\cos \left(G_{y} a / 2\right)$. The band structure for 2D honey comb lattice with circular and hexagonal GaAs dielectric rods of radius $0.23 a$ and width $w=0.3 a$ respectively are shown in Figure 14.

A complete band gap for circular GaAs rods was observed between the seventh and eight TM bands and between the fifth and the sixth TE bands. The mid gap ratio is $6 \%$ while the width of the complete gap is $0.43(\omega a / 2 \pi c)$. For hexagonal GaAs rods, a complete band gap for both polarizations was observed between the sixth and seventh TM bands and third and fourth TE bands. The photonic gap width is $0.069(\omega a / 2 \pi c)$ around a mid gap frequency $0.3(\omega a / 2 \pi c)$. Gap to mid gap ratio is $20.91 \%$.

The dielectric contrast and the filling factor for honeycomb lattice with circular dielectric rods and hexagonal dielectric rods in air are shown in Figure 15. A maximum value for the gap width to mid gap frequency ratio exist around $\varepsilon=15$.

The minimum dielectric contrast needed (7.5) to open a complete band gap was slightly higher than that for a triangular lattice. The largest gap occurs for a filling fraction of 0.12 . The range of filling fraction for GaAs rods for which a complete gap could be observed is limited. The minimum dielectric index contrast to open a gap and the filling factor for the highest gap for the different photonic structures are tabulated in Table 2.

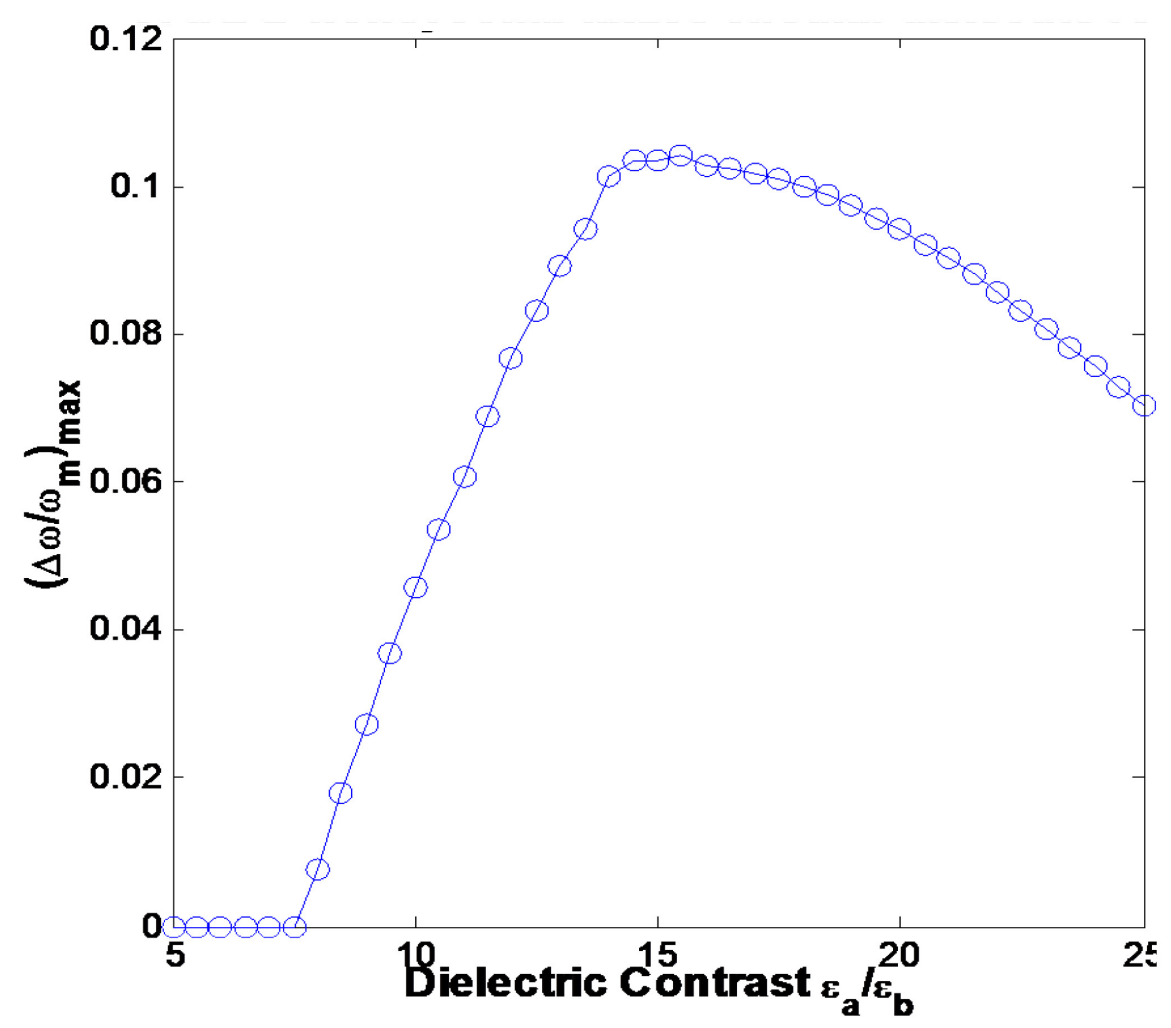

$15(a)$ 


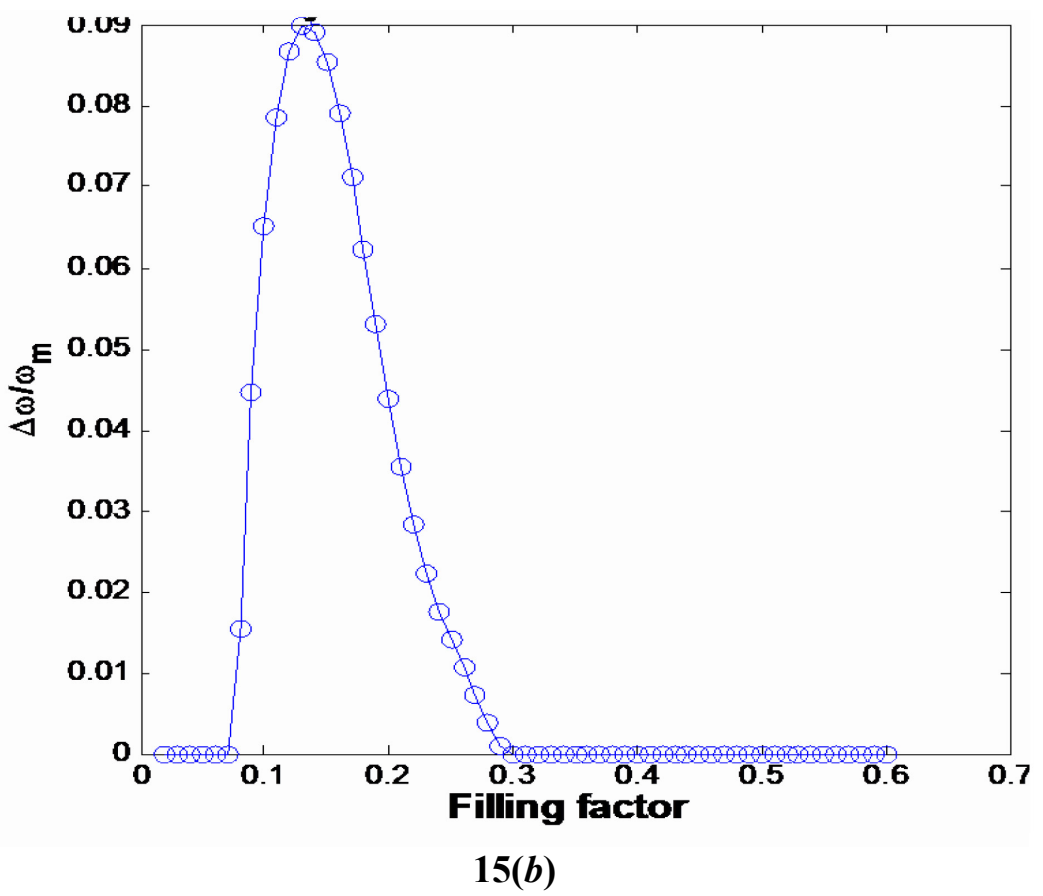

Fig. 15. Variation of maximum complete band gap width to mid gap ratio with (a) dielectric contrast and $(b)$ filling factor for honeycomb lattice with circular dielectric rods.

Table 2. The minimum dielectric index contrast to open a photonic gap and the filling factor for highest gap for different photonic structures.

\begin{tabular}{|c|c|c|c|}
\hline $\begin{array}{l}\text { Crystal } \\
\text { lattice }\end{array}$ & Structure & $\begin{array}{l}\text { Minimum dielectric } \\
\text { index contrast to } \\
\text { open a photonic gap }\end{array}$ & $\begin{array}{l}\text { Filling factor and its value } \\
\text { for highest gap }\end{array}$ \\
\hline \multirow[t]{4}{*}{ Square } & Square rods in air & $3(\mathrm{TM})$ & $f=w^{2} a^{-2}, 0.13\left(\varepsilon_{a}=13\right)$ \\
\hline & Circular rods in Air & $3(\mathrm{TM})$ & $f=\pi r^{2} a^{-2}, 0.13\left(\varepsilon_{a}=13\right)$ \\
\hline & Square air holes & 13 & $0.82\left(\varepsilon_{b}=18\right)$ \\
\hline & Circular air holes & 7.5 & $0.76\left(\varepsilon_{b}=18\right)$ \\
\hline \multirow[t]{4}{*}{ Triangular } & Circular rods in Air & 2 & $\begin{array}{c}f=2 \pi r^{2} / \sqrt{3} a^{2}, 0.13 \\
\left(\varepsilon_{a}=13\right)\end{array}$ \\
\hline & $\begin{array}{l}\text { Hexagonal rods in } \\
\text { Air }\end{array}$ & 2 & $f=6 w^{2} a^{-2}, 0.10\left(\varepsilon_{a}=13\right)$ \\
\hline & Circular air holes & 7 & $0.84\left(\varepsilon_{b}=13\right)$ \\
\hline & Hexagonal air holes & 7 & $0.75\left(\varepsilon_{b}=13\right)$ \\
\hline Hexagonal & Circular rods in Air & 7.5 & $\begin{array}{c}f=4 \pi r^{2} / 3 \sqrt{3} a^{2}, 0.12 \\
\left(\varepsilon_{a}=13\right)\end{array}$ \\
\hline
\end{tabular}




\section{GAP MAPS}

The gap map which are particularly useful in engineering possibilities of photonic crystals, were calculated for GaAs dielectric rods in air and air holes in GaAs for all configurations varying the radius or the width of the rods or air holes by an iterating procedure using the plane wave expansion method for the first 20 bands. The gap maps indicate the location of the gaps for a particular GaAs lattice. For the square lattice of square and circular GaAs rods as well as circular air holes in GaAs, the gap maps are presented in figure 16 with the complete band gaps in green, TM gaps in red and TE gaps in blue.

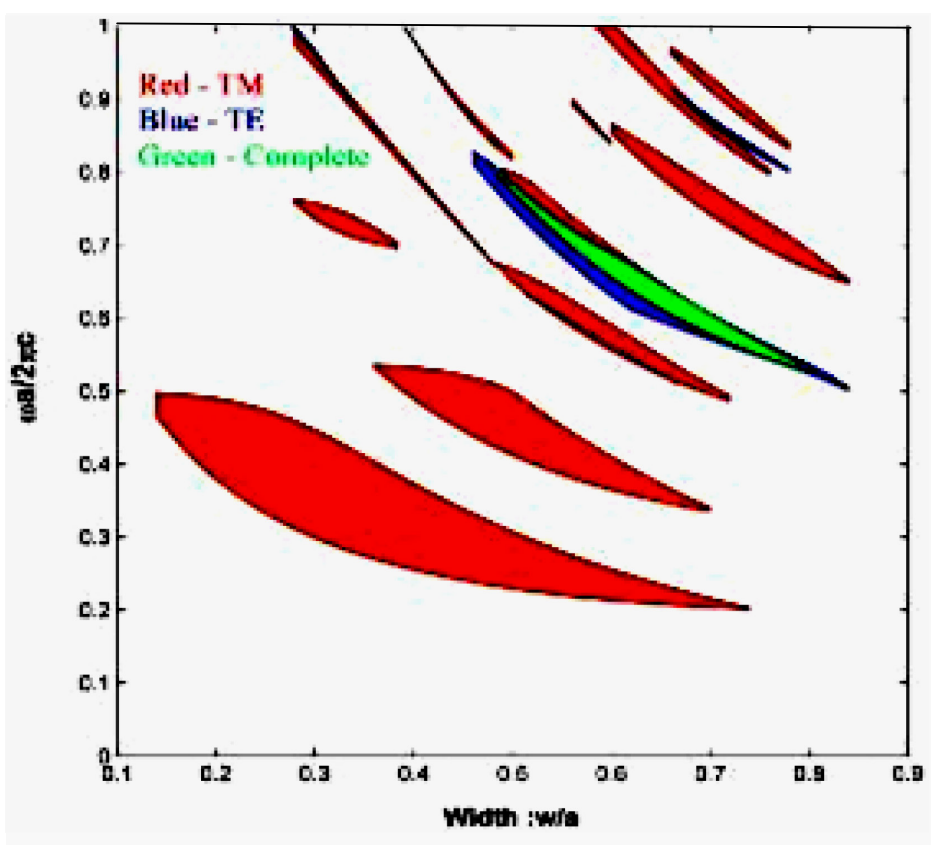

$16(a)$

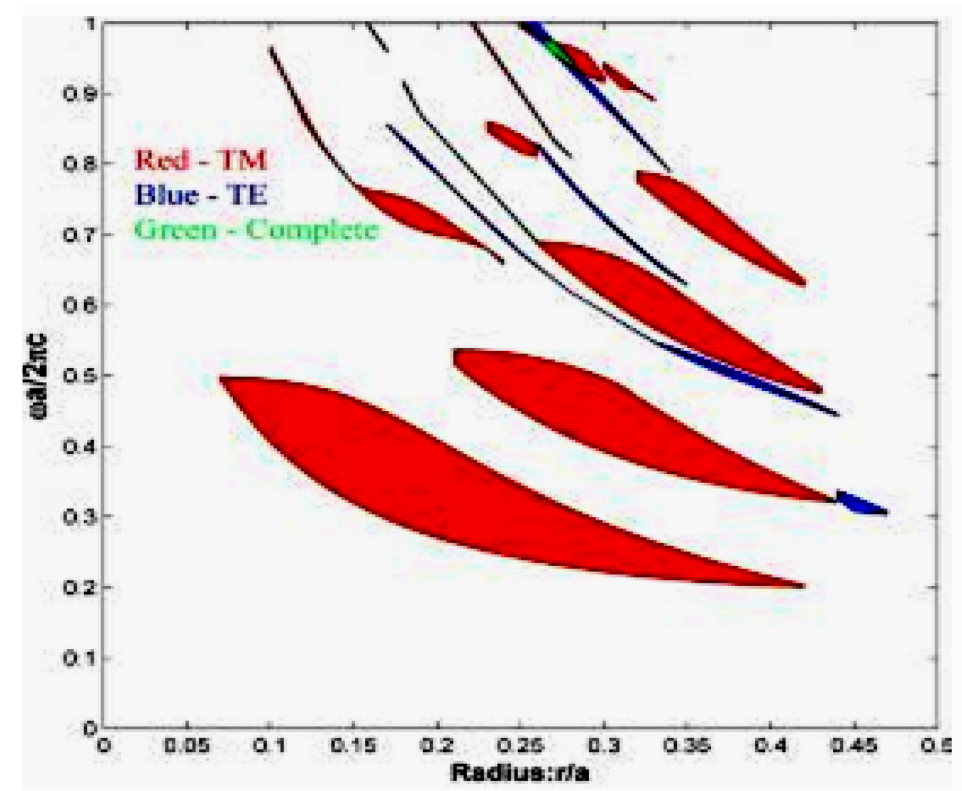

16(b) 


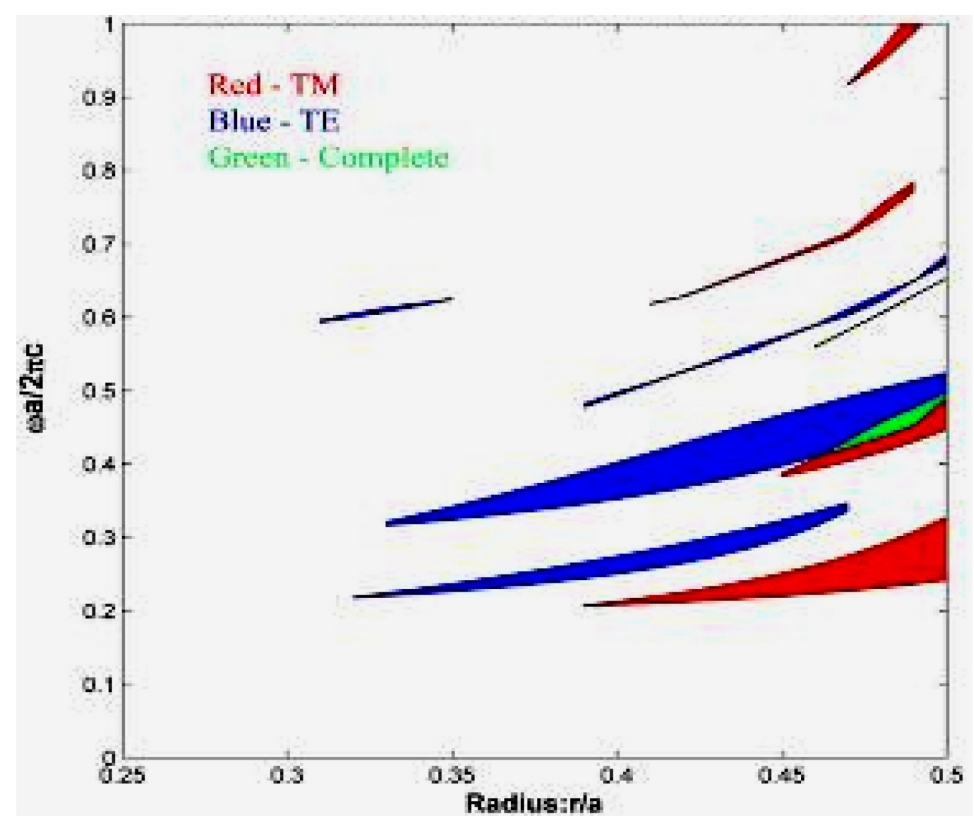

$16(c)$

Fig. 16. Gap map for square lattice of $(a)$ square GaAs rods, $(a)$ circular GaAs rods and (c) circular air holes in GaAs.

TM gaps are more favoured for dielectric rods while TE gaps are more favoured for air holes. For square lattice of square GaAs rods for $w / a \sim 0.5-0.8$ and of circular air holes in GaAs for $r / a \sim 0.42-0.50$ photonic gaps were observed. In Figure 17 the gap maps for triangular lattices of circular and hexagonal GaAs rods in air and air holes in GaAs are presented. Complete band gaps were obtained for all the types of triangular lattices. For dielectric rods, except at higher frequencies for $r / a$ larger than 3.5 the patterns are similar.

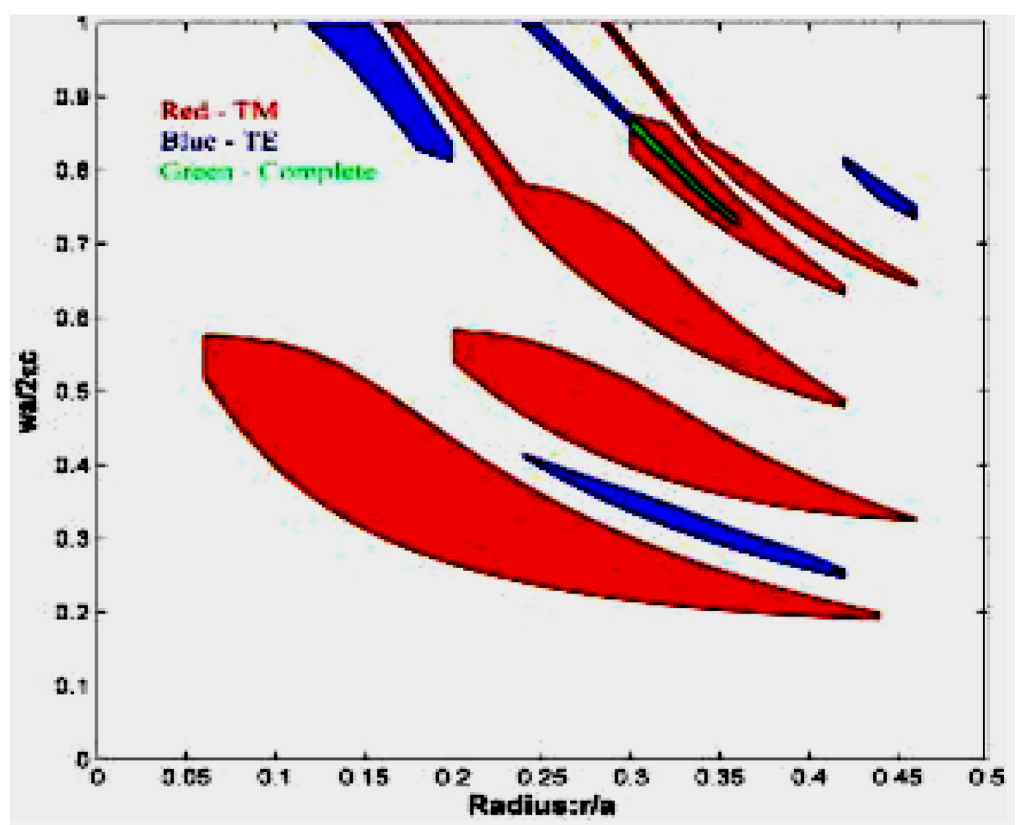

$17(a)$ 


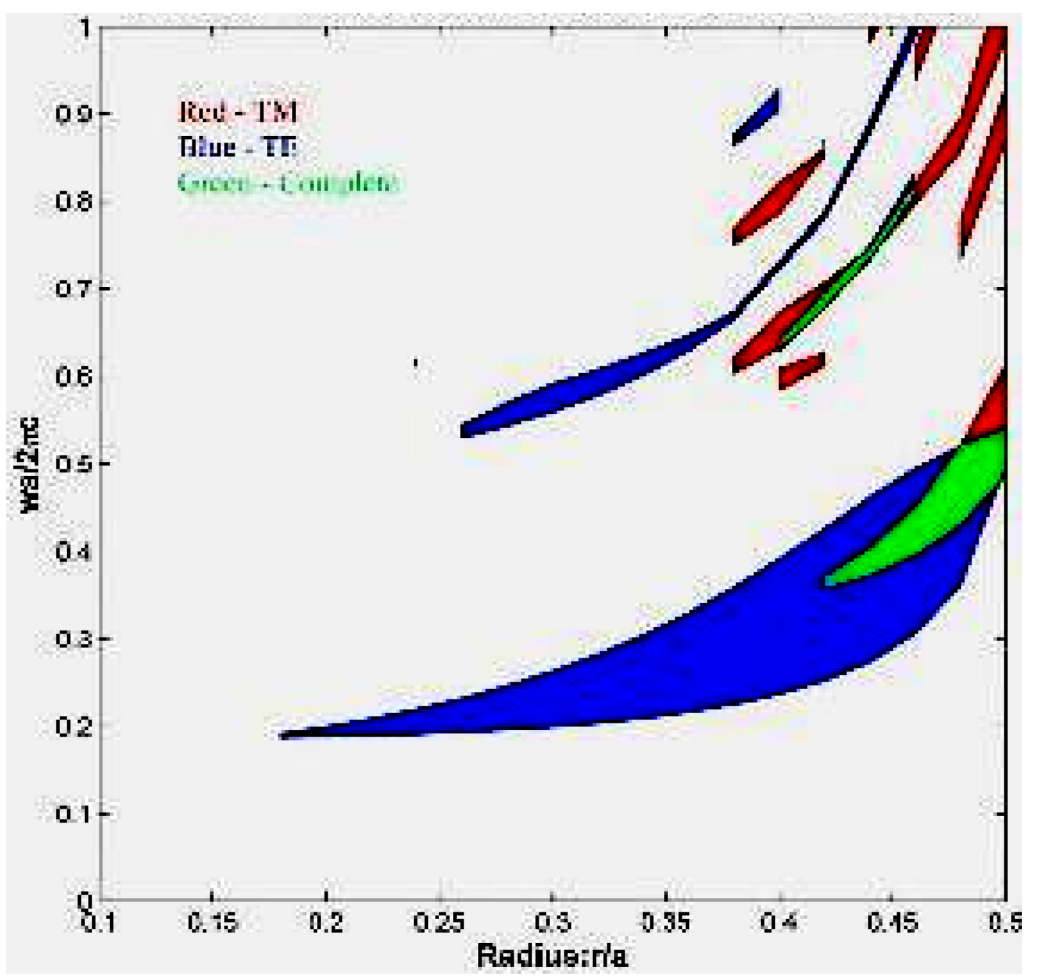

17(b)

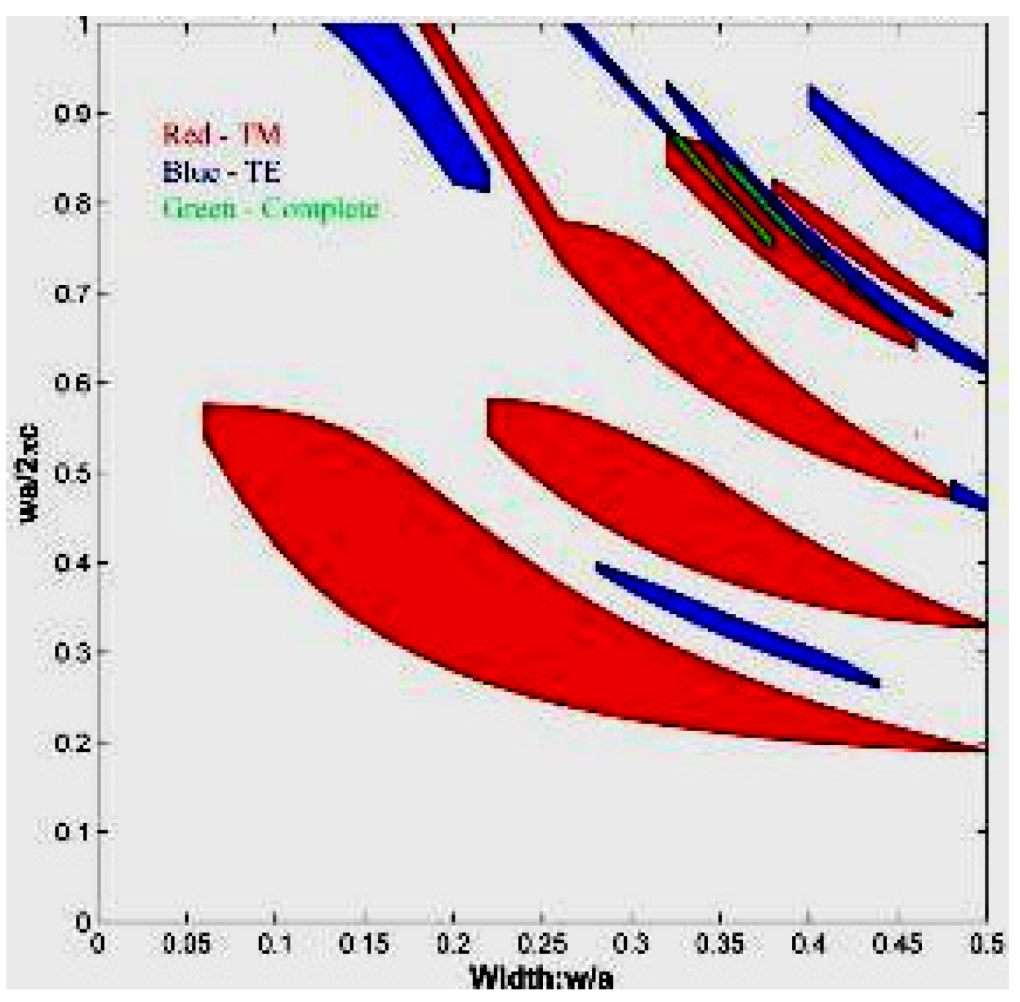

17(c) 


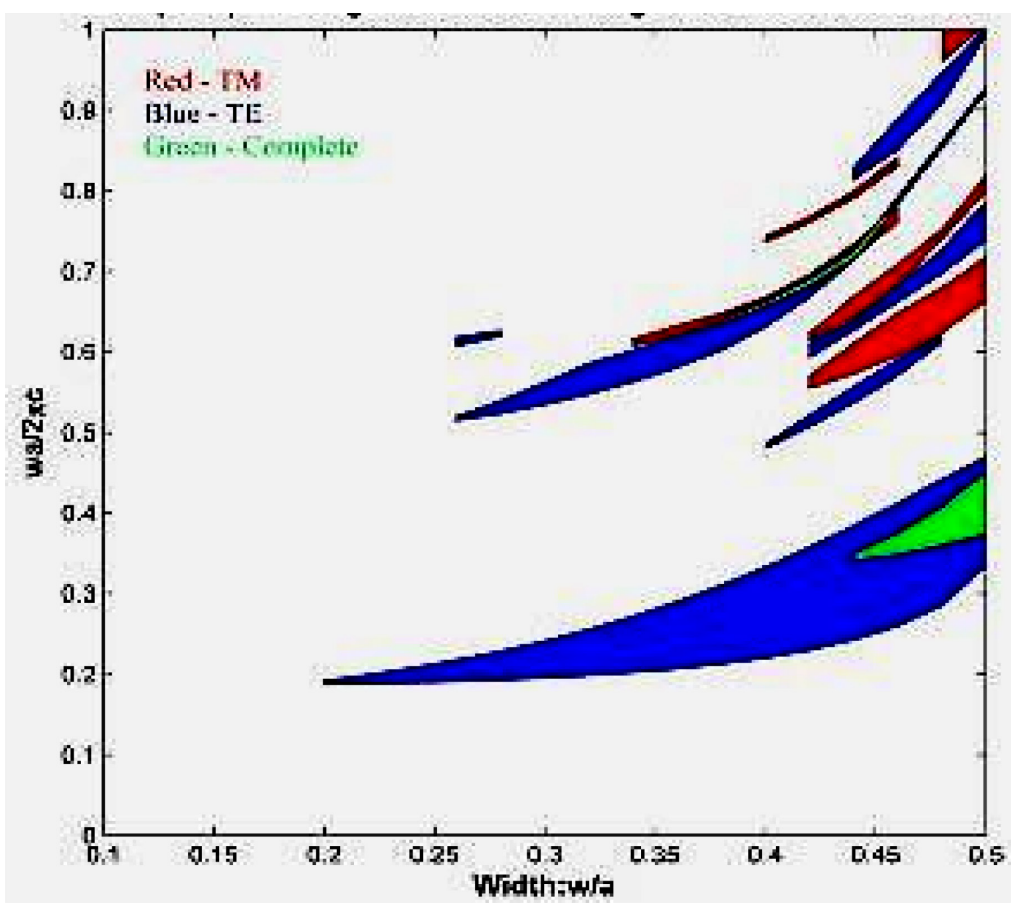

$17(d)$

Fig. 17. Gap maps for triangular lattice of (a) circular GaAs rods in air, (b) circular air holes in GaAs, (c) hexagonal GaAs rods in air, d) hexagonal air holes in GaAs. Red-TM, blue-TE and greencomplete gap.

The gap map for honeycomb lattices of circular and hexagonal GaAs rods in air as well as circular alumina rods with lower dielectric constant of 9 in air are shown in Figure 18.

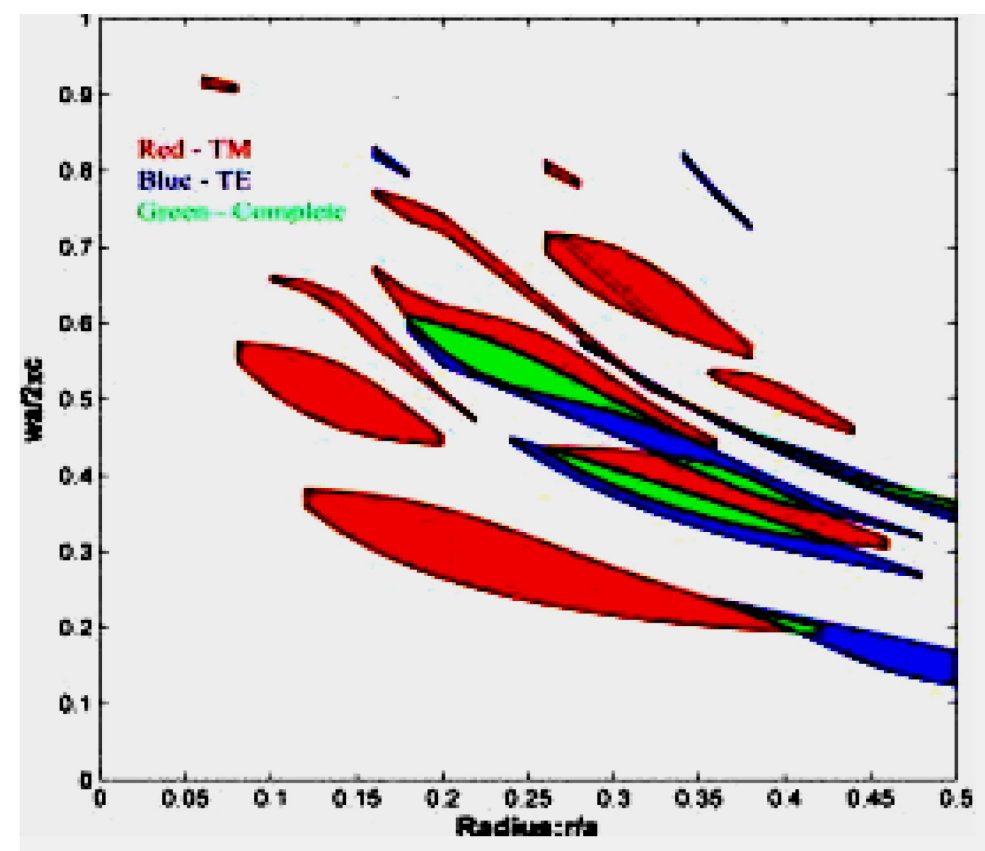



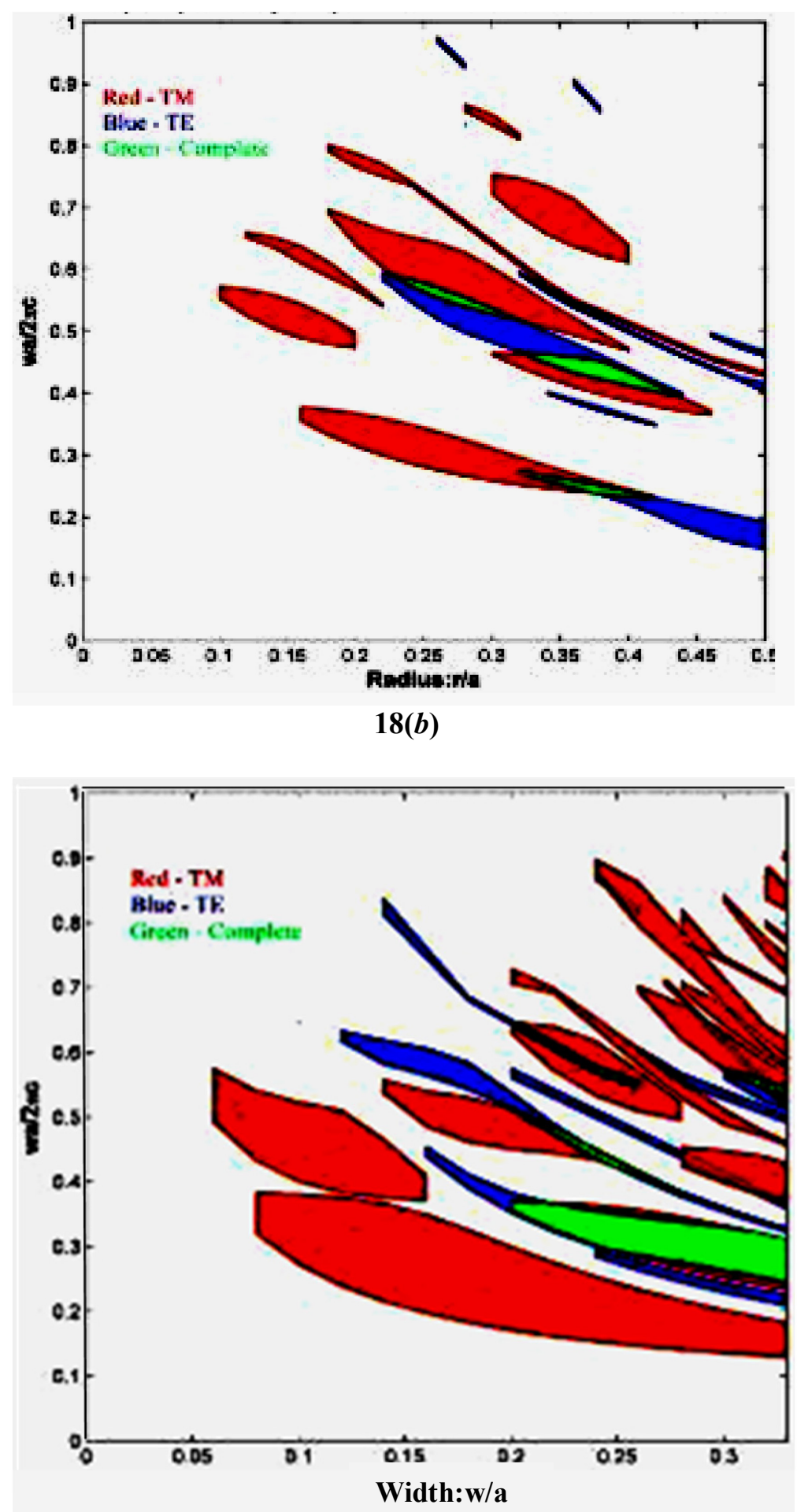

$18(c)$

Fig. 18. Gap maps for honeycomb lattice of $(a)$ circular GaAs rods in air, $b$ ) circular alumina $\operatorname{rods}(\varepsilon=9)$ in air and $(c)$ hexagonal GaAs rods in air. Red-TM, blue-TE and green-complete gap.

The gap maps of circular and hexagonal GaAs rods show a quite large difference. Both kinds of rods give rise to formation of complete band gaps. The circular rods give large complete band gaps for higher frequencies while hexagonal rods give a complete band gap at low frequencies. However band diagram of hexagonal cross sectional rods are more packed at 
lower frequencies than that of circular rods. The circular alumina rods gave a smaller complete gap width than GaAs rods while for circular air holes in GaAs, a complete gap was not observed.

\section{GEOMETRICAL GAP MAPS}

The lattice constant and the radius or widths of the rods were varied in a parallel algorithm in search of a band gap around the telecommunication wavelength $\lambda \sim 1.5 \mu \mathrm{m}$ region. Geometrical gap maps, appropriate for a square lattice with circular and square GaAs rods and air holes in GaAs are shown in Figure 19.

The TM gap widths and the mid gaps in terms of wavelength are presented in Figure 19(a) and (b) for circular and square GaAs rods respectively. The dots indicate that the band gap for corresponding physical parameters lying around $\lambda=1.55 \mu \mathrm{m}$.

For a point inside this area, the photonic crystal has a band gap for this wavelength at these geometrical parameters. About 5000 iterations were done to achieve this kind of a diagram. According to the figures, the photonic crystal of square dielectric rods has a wider gap map than of circular rods for TM mode.

The mid gap wavelength is indicated as a dot while the gap width is indicated by vertical lines for selected points in the map. The geometrical gap maps for TE modes of a square lattice with both circular and square cross sectional air holes in GaAs are shown in the Figure $19(c)$ and $(d)$. These gap maps are narrower than the gap maps obtained for TM modes of GaAs rods.
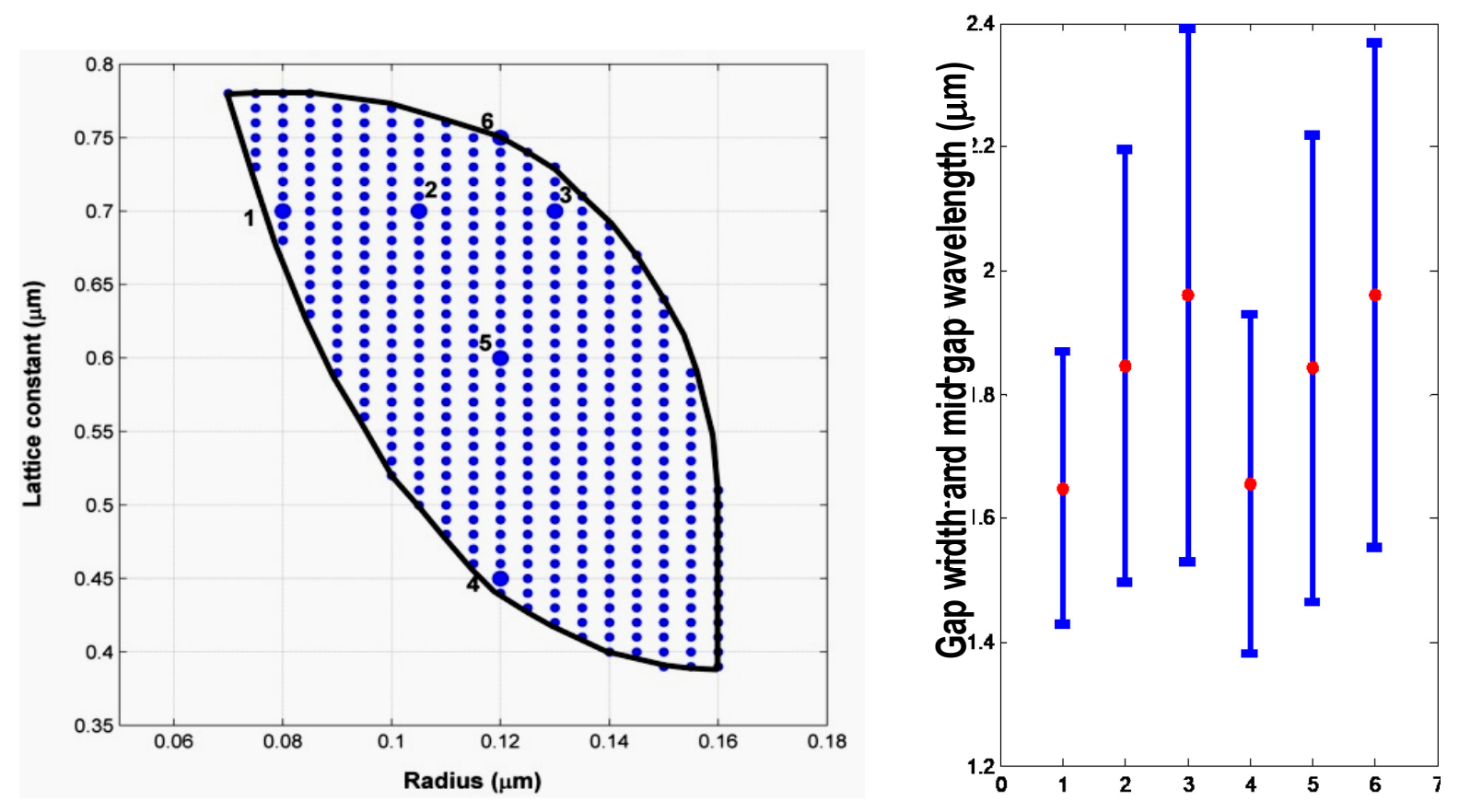

19(a) 

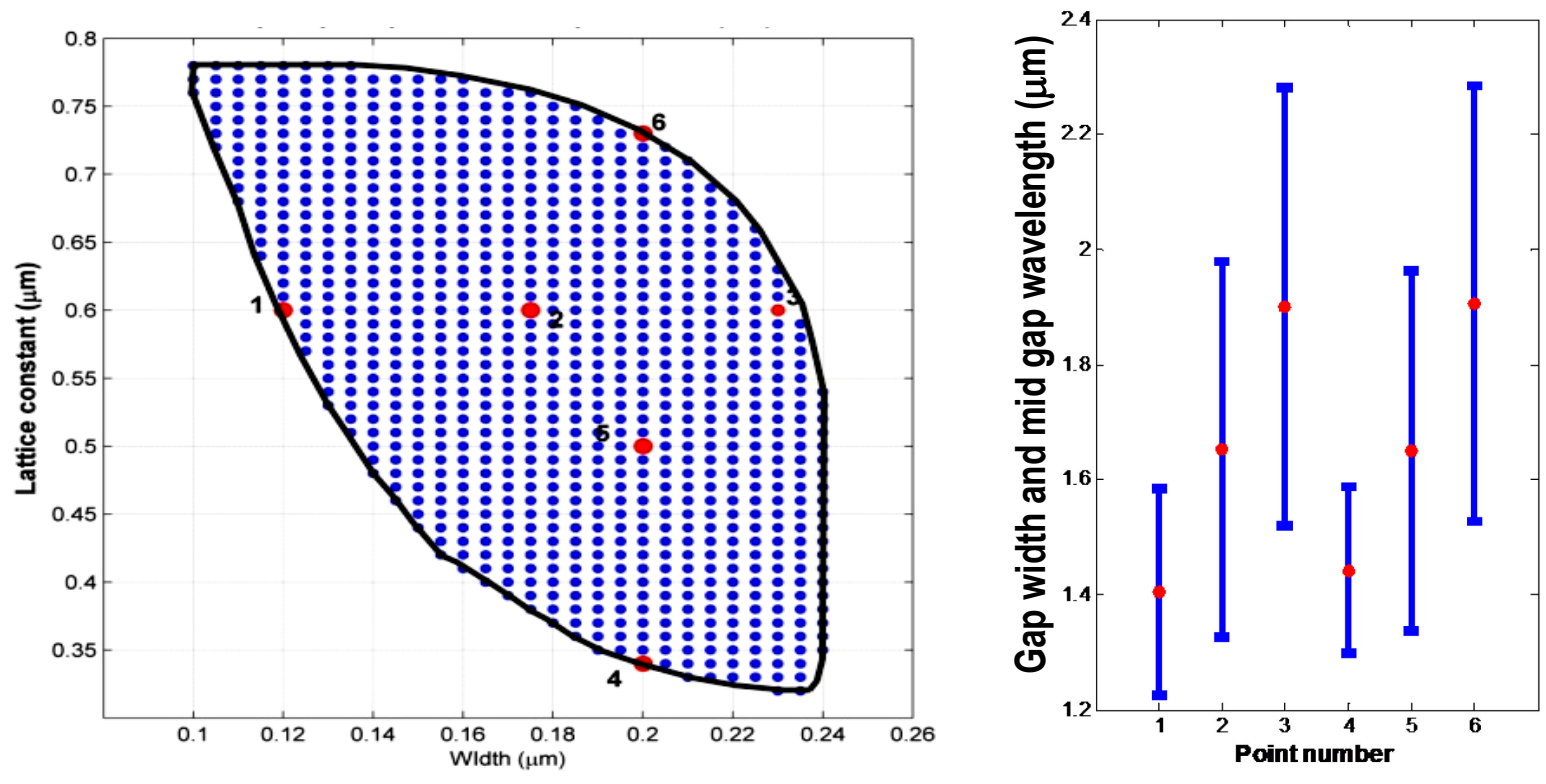

19(b)

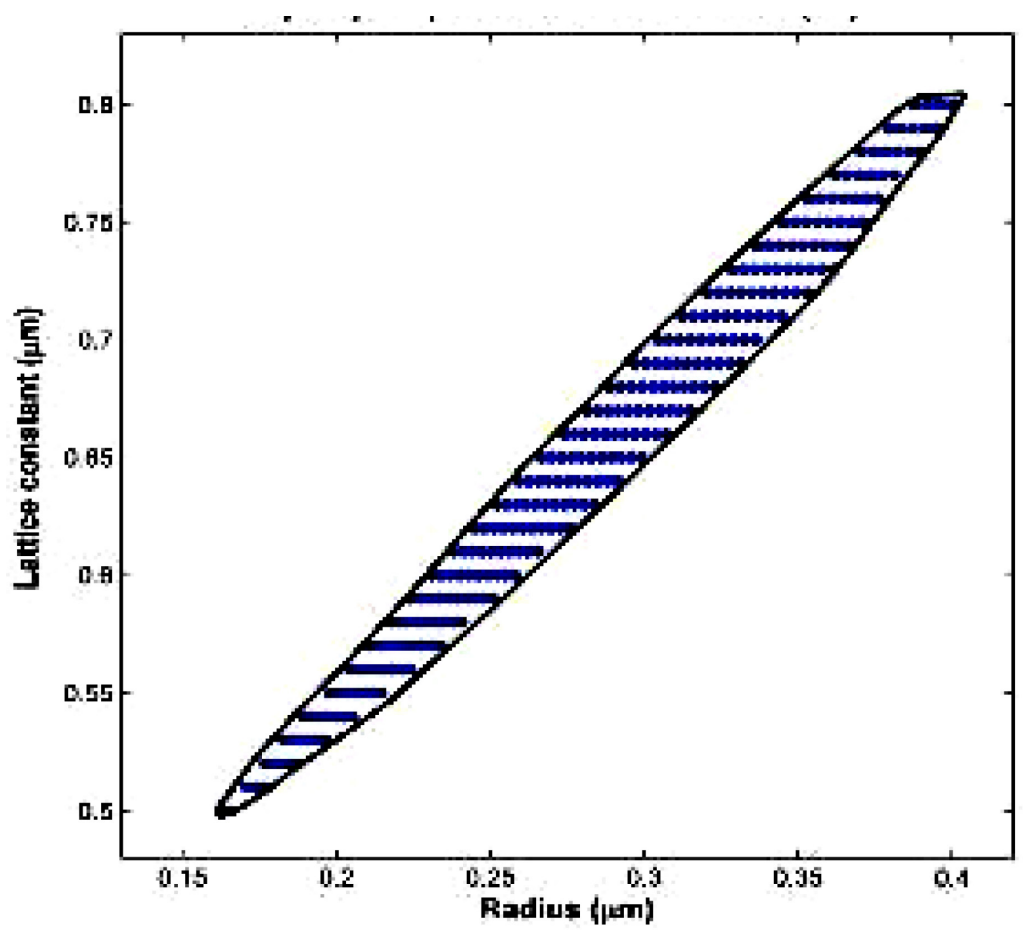

19(c) 


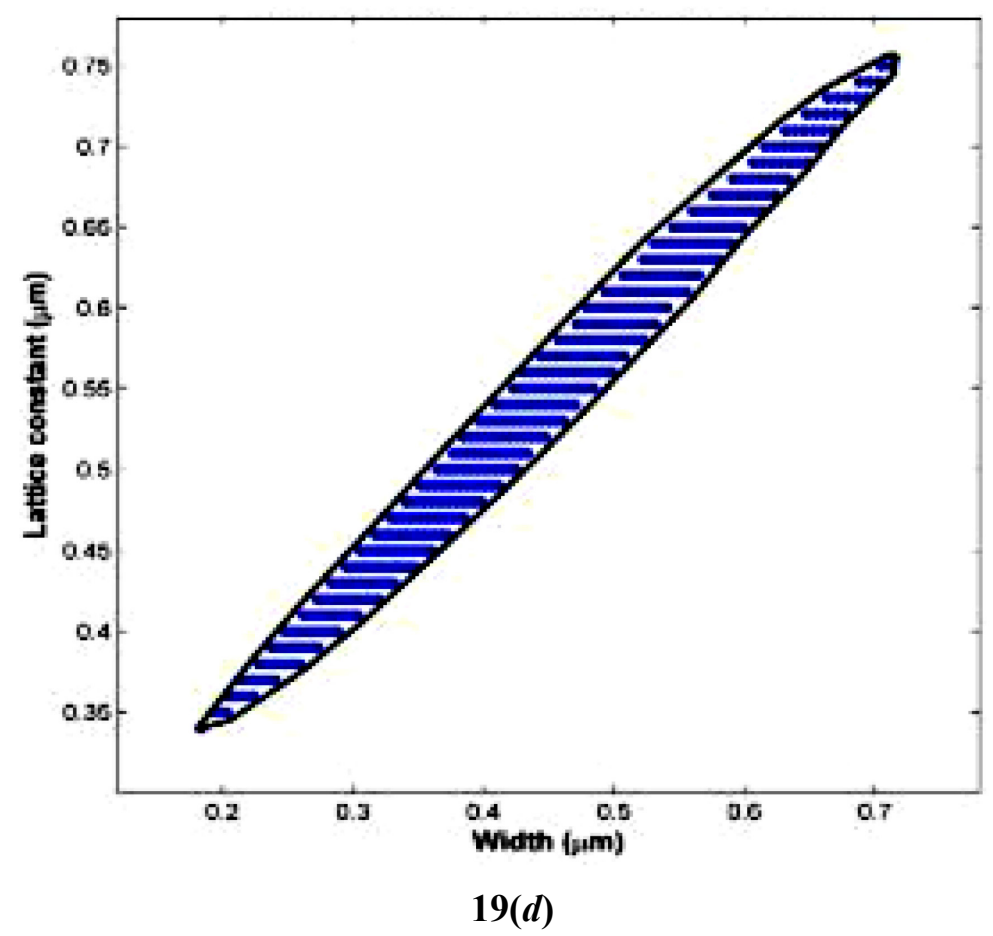

Fig. 19. Geometrical gap map around $\lambda=1.55 \mu \mathrm{m}$ for square lattice with $(a)$ circular, $(b)$ square GaAs rods in air for TM mode and gap width (left) and central values for selected points in the gap map (right). Gap maps of (c) circular, (d) square air hole in GaAs for TE mode.

The appropriate geometrical gap maps for triangular lattice with circular and hexagonal holes drilled in GaAs for operation around telecommunication wavelength are presented in Figure 20.

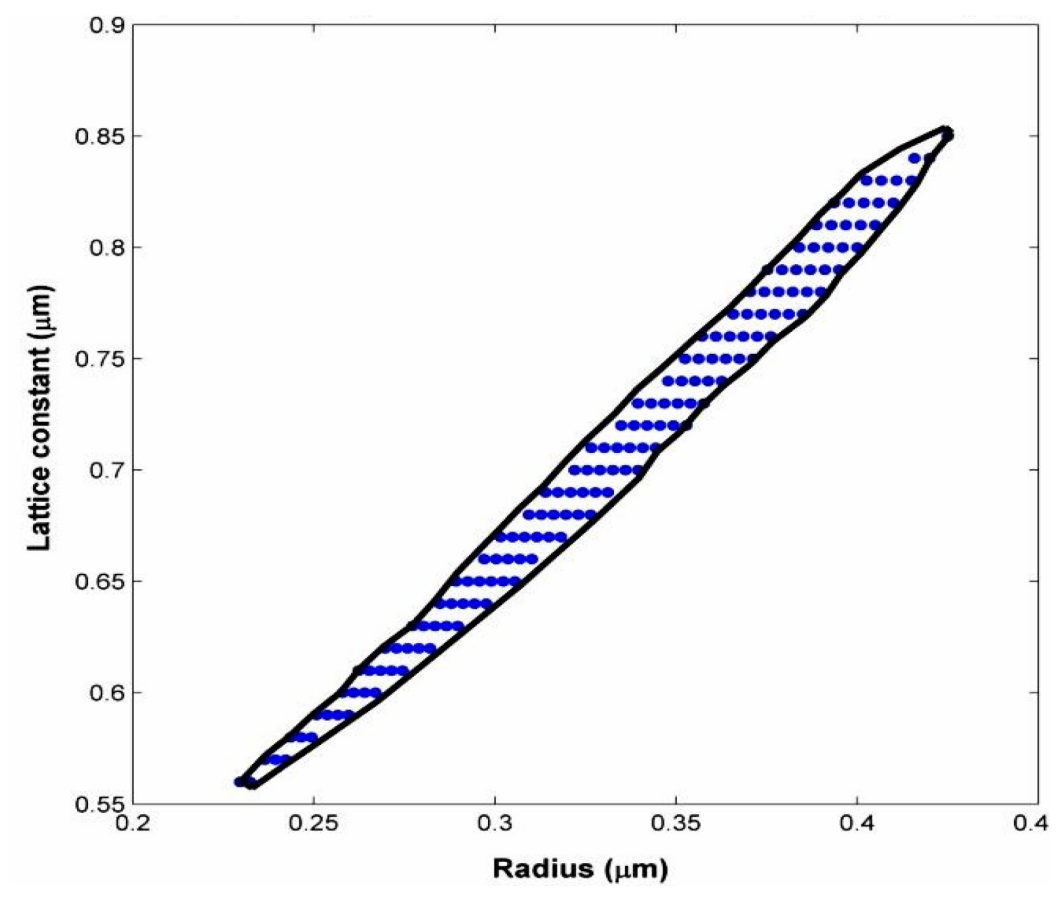

20(a) 


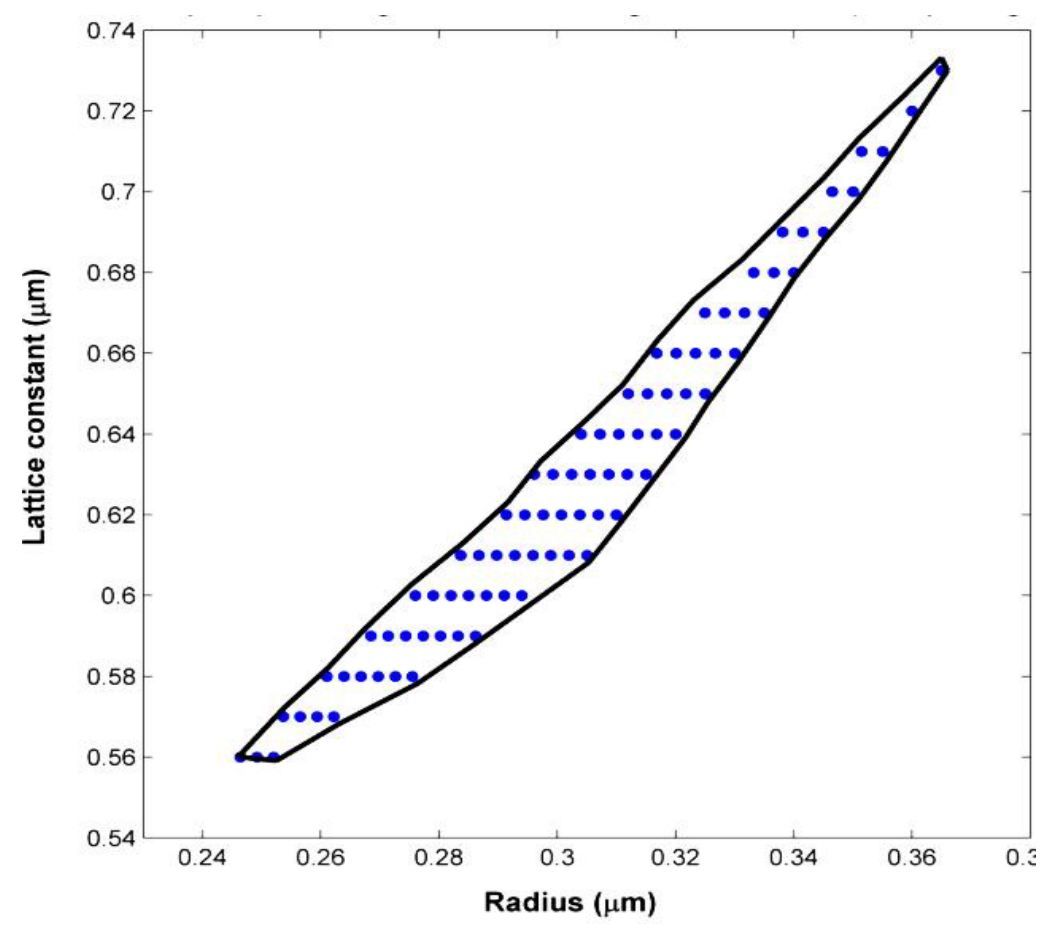

20(b)

Fig. 20. Geometrical gap map around $\lambda=1.55 \mu \mathrm{m}$ for triangular lattice with $(a)$ circular air holes and (b) hexagonal air holes in a dielectric medium of $\varepsilon=13$.
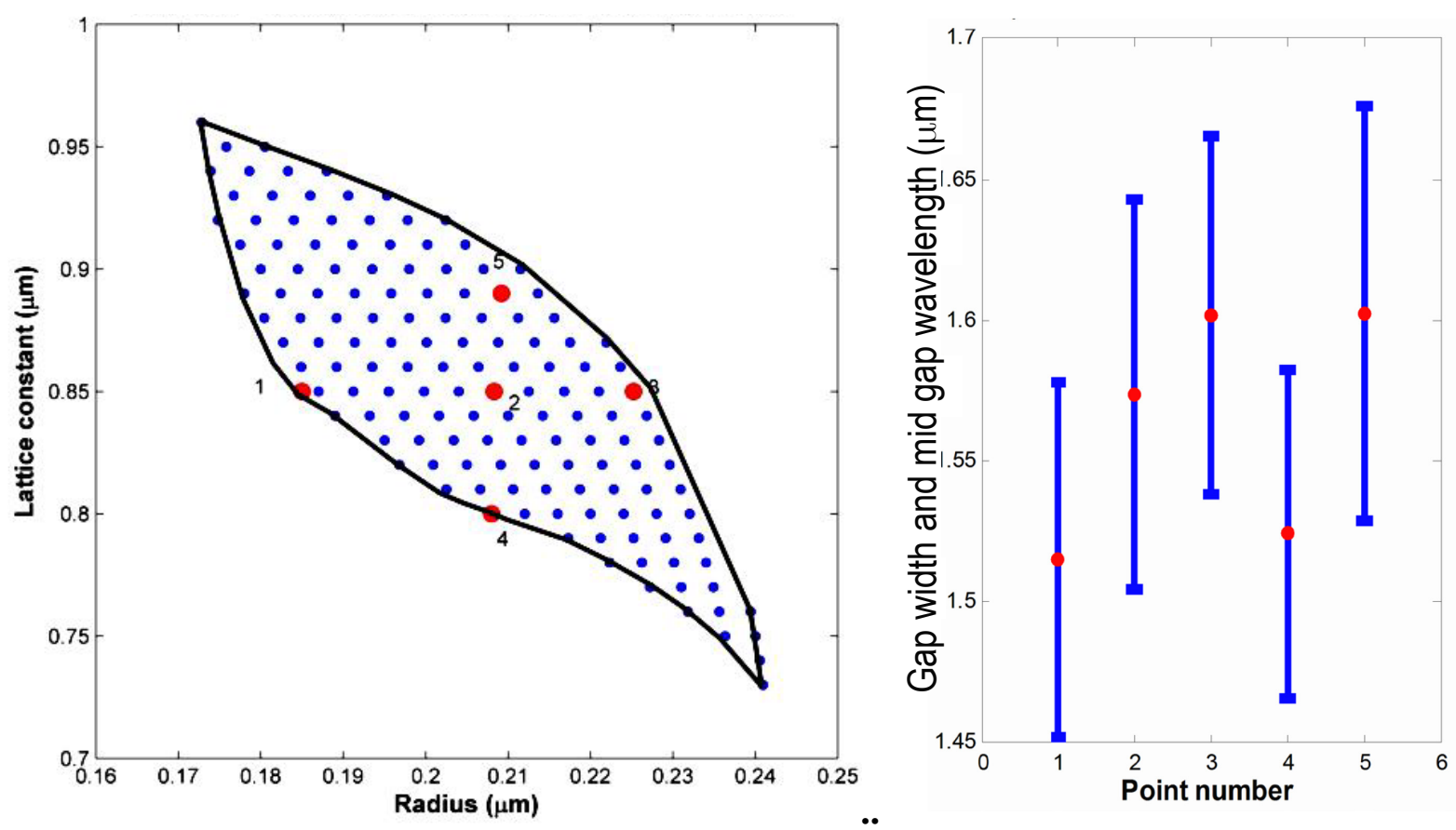

Fig. 21. Geometrical gap map appropriate for operation around $\lambda=1.55 \mu \mathrm{m}$ for honeycomb lattice with circular dielectric rods for $\varepsilon=13$. Gap map (left) and gap width and central values for selected points in the gap map (right).

The appropriate geometrical gap maps for triangular lattice with circular and hexagonal holes drilled in GaAs for operation around telecommunication wavelength are presented in Figure 20. Complete gap around $\lambda=1.55 \mu \mathrm{m}$ could be achieved by selecting lattice constant 
and radius within the area specified by the thick lines. About 3500 iterations were done in order to find a correct shape of the map. At telecommunication wavelengths they have complete band gaps for both polarizations. These maps give the correct geometrical parameters for designing applications like filters and wave guides.

The geometrical gap map calculated for a honeycomb lattice formed from circular GaAs rods in air has a complete gap around $\lambda=1.55 \mu \mathrm{m}$ (Figure 21). Therefore a photonic band gap can be obtained for honeycomb lattice of isolated dielectric rods, at high frequencies. This could even be tuned to operate near-infrared wavelengths. As expected, it showed a larger gap comparable to other lattices.

\section{CONCLUSIONS}

The dielectric constants and filling fractions are important parameters and mode field distribution not only depended on geometries but greatly characterized by the cross section of the rods and the shape of the localized medium used. The refractive index contrast must be high enough to have a considerable band gap. A large TM band gap was observed for square lattice with circular dielectric rods with small filling fractions. But fabricating a photonic crystal with small rods about $100 \mathrm{~nm}$ of radius in air or in a medium with small dielectric constant is challenging. The square lattice of square dielectric rods has a complete band gap at high frequencies.

The variation of TM gap to mid gap ratio with dielectric contrast revealed that square lattice of circular rods require a lower dielectric contrast to generate a TM band gap than square rods. The array of square rods gives rise to a larger TM band gap when the dielectric contrast is greater than 16.3. A photonic gap can be obtained for square lattice of air holes for both square and circular cross sections for a dielectric contrast of 18. For square lattice, square GaAs rods in air gave a wider photonic gap than circular air holes in GaAs. Triangular lattice is the only simple geometry that could give rise to a complete band gap between the lower bands.

Circular and hexagonal dielectric rods needed the same minimum dielectric contrast to open a photonic band gap. A maximum value for the gap width to mid gap frequency ratio occurs for a dielectric contrast of 15. For higher dielectric constants, the circular holes yield a larger gap than the hexagonal holes. Circular and hexagonal air holes in GaAs gave wider gap maps than circular and hexagonal GaAs rods in air. Honeycomb lattice behaved differently compared to other lattices. A complete gap can be achieved for honeycomb lattice of circular and hexagonal dielectric rods. These gaps had no correlations.

The position of the gaps and filling ratio had a significant difference between these two rods. For circular dielectric rods, the photonic gaps become wider with increase dielectric constant but hexagonal GaAs rods gave a wider photonic gap than circular GaAs rods. Air holes in GaAs did not create any significant gap for both TM and TE modes. The geometrical gap maps of GaAs material showed that, a complete band gap can be achieved for honeycomb lattice of isolated dielectric rods, at high frequencies. 


\section{References}

[1] J. W. S. Rayleigh, Phil. Mag. 26 (1988) 256-265.

[2] V. P. Bykov, Sov. Phys. JETP 35 (1972) 269-273.

[3] E. Yablonovich, Phys. Rev. Lett. 58 (1987) 2059-2062.

[4] S. John, Phys. Rev. Lett. 58 (1987) 2486-2489.

[5] K. M. Ho, C. T Chang, C. M. Soukoulis, Phys. Rev. Lett. 65 (1990) 3152-3155.

[6] S. C. Johnson, J. D. Joannopoulos, Opt. Express 8 (2001) 173-190; J. D. Joannopolus, S.C. Johnson J.N. Winn, R.D. Meade, Photonic Crystals: Modeling the flow of light, Princeton University Press, (2008) Chapter 5, p. 66-72.

[7] K. Skoda, Phys. Rev. B. 52 (1995) 8992-9002.

[8] D. Labilloy D. Labilloy, H. Benisty, C. Weisbuch, T. F Krauss, R. M. De La Rue, V. Bardinal, R. Houdré, U. Oesterle, D. Cassagne, C. Jouanin, Phys. Rev. Lett. 79 (1997) 4147-4150.

[9] H. Benisty, C. Weisbuch, D. Labilloy, M. Rattier, C. J. M. Smith, T. F. Krauss, R. M De La Rue, R. Houdré, U. Oesterle, C. Jouanin, D.Cassagne, Journal of Lightwave Technology 17 (1999) 2063-2076.

[10] M. Philal, A. A. Maradudin, Phys. Rev. B 44 (1991) 8565-8571.

[11] P. R. Villenuve, M. Piche, Phys. Rev. B 46 (1992) 4973-4975.

[12] Pi-Gang Luan, Zhen Ye, Two Dimensional Photonic crystals (2001), arXiv: condmat/0105428.0. 